\title{
REMATE DE DERECHOS DE AGUA EN CHILE: ¿QUÉ PASÓ POSREFORMA DEL CÓDIGO DE AGUAS DE 2005?*
}

\author{
Julio Peña-Torres \\ Pontificia Universidad Católica de Chile, Chile \\ Emiliano Vargas \\ Ministerio de Obras Públicas, Chile \\ Guillermo Donoso \\ Pontificia Universidad Católica de Chile, Chile
}

\begin{abstract}
RESUMEN: Este es el primer análisis sobre el uso de remates para la asignación inicial de derechos de agua en Chile. Con datos para 2005-2018, se analizan los efectos de la reforma del año 2005 del Código de Aguas sobre el uso de remates. Posreforma, una proporción mínima de los nuevos derechos otorgados fueron asignados vía remates; $57 \%$ de los remates convocados se declararon desiertos. En los remates que ocurrieron, con frecuencia asistieron pocos participantes. $\mathrm{Y}$ en los remates con pocos participantes predominaron
\end{abstract}

Julio Peña-Torres es Ph.D. en Economía. Investigador CLAPES UC, Pontificia Universidad Católica de Chile. E-mail: julioalept@gmail.com. Autor para correspondencia.

Emiliano Vargas es Magíster en Economía. Ministerio de Obras Públicas, Chile.

Guillermo Donoso es Ph.D. en Economía Agraria. Profesor titular, Facultad de Agronomía e Ingeniería Forestal, y Centro de Derecho y Gestión de Aguas, Pontificia Universidad Católica de Chile.

* Se agradece la información provista por Manuel Madrid (ENACON S.A.), Francisco Puelma (Dirección General de Aguas) y Fernando Fuentes (Universidad Alberto Hurtado). También se agradecen los valiosos comentarios de Ricardo Paredes y de dos referees anónimos. 
adjudicaciones a los precios mínimos de oferta. Se analizan hipótesis y evidencia sobre estos hechos. En tanto sea válido el objetivo de dinamizar el uso de licitaciones para asignar nuevos derechos de agua, se plantean desafíos por resolver.

Palabras Clave: remate, subastas multiobjeto, derechos de agua, Código de Aguas, licitación, derechos de producción

RECIBIDO: septiembre 2018 / ACEPTADO: junio 2019

\section{ORAL AUCTIONS OF WATER USER RIGHTS IN CHILE: WHAT HAPPENED POST REFORM OF THE WATER CODE IN 2005?}

ABSTRACT: We carry out the first analysis on the use of oral priceascending auctions to allocate water user rights in Chile. With data from 2005 to 2018, the effects of the 2005-reform of the Water Code on the use of auctions are analyzed. Following the reform, a minimum proportion of the new rights granted were allocated via auction. In fact, $57 \%$ of the called auctions were declared void. On the other hand, in the auctions that did take place, often a few participants attended. Moreover, in those auctions with few participants, awards at the minimum bid prices stood out. Hypotheses and evidence on these facts are analyzed. As long as the objective of invigorating the use of auctions to award new water rights remains valid, we pinpoint challenges to be resolved.

KEYwords: auction, multiobject auctions, water user rights, Chilean Water Code, public tendering, production rights

ReCEIVED: September 2018 / ACCEPTED: June 2019

\section{INTRODUCCIÓN}

- egún la ley chilena, cuando existe rivalidad entre solicitudes por un nuevo derecho de aprovechamiento de agua (DAA), la asignación debe realizarse mediante remate al mayor precio ofertado (Código de Aguas, Arts. 142 y 145). La opción de usar remates ya existía en el Código de Aguas (CA, en adelante) de 1981 pero, previo a la reforma del CA realizada en 2005, raramente se usó (Gentes 2006). En el debate legislativo previo a la reforma de 2005, se argumentó que la Dirección General de Aguas (DGA, ente regulador de la asignación de DAA), en vez de recurrir al mecanismo de remates, había "usado mecanismos bu- 
rocráticos para solucionar dicha escasez [solicitudes de DAA en disputa], suspendiendo o dilatando la concesión sin dejar que el mercado actúe" (Congreso Nacional 2005).

En el debate de entonces se argumentó que (i) existían porcentajes relevantes de DAA no consuntivos asignados, que estaban sin uso ${ }^{1}$ y (ii) que esto era consecuencia de estrategias disuasorias de entrada, acumulando titularidad y nuevas solicitudes de DAA en manos de los principales incumbentes en el mercado de generación hidroeléctrica, para evitar la entrada de rivales en generación eléctrica (Peña, Luraschi y Valenzuela 2004; TDLC 2007). Entre los impulsores de la reforma de 2005 prevalecía el convencimiento de que la entrega gratuita de DAA, junto con no cobrar por el 'no uso' de DAA otorgados, eran causas sustantivas de estos problemas.

La reforma de 2005 incorporó cambios que, aparte de introducir el cobro de una patente por no uso de DAA otorgados, buscaban privilegiar el uso del remate como mecanismo predominante de asignación de nuevos DAA. Para estos efectos, la reforma introdujo un conjunto de cambios procedimentales relativos a la opción del uso de remates. ${ }^{2}$ El objetivo central de este trabajo es analizar qué pasó, posreforma de 2005, con el uso del remate como mecanismo asignador de nuevos DAA, identificando razones de lo ocurrido.

Este es el primer análisis sobre el uso de remates para la asignación inicial de DAA en Chile. ${ }^{3}$ Con datos desde 2005 a 2018, se analizan los efectos sobre el uso de remates, así como de los cambios incorporados en la reforma de 2005 al Código de Aguas (CA). Contrario a lo esperado, una proporción mínima (menos del 1\%) de los nuevos caudales otorgados durante 2005-2018 fueron asignados vía remate. Durante los 14 años analizados ocurrieron pocos remates de DAA (134 en total).

\footnotetext{
${ }^{1}$ Según Riestra (2008), de un total de $15.000 \mathrm{~m}^{3} / \mathrm{s}$ concedidos en DAA no consuntivos hacia fines de 2005 , solo $18,6 \%$ de ellos eran utilizados.

${ }^{2} \mathrm{La}$ reforma no introdujo cambios institucionales ni mejoras presupuestarias al interior de la DGA. Tampoco introdujo cambios en el mecanismo de remate, ni en las metodologías de cálculo e información sobre los precios mínimos de oferta.

${ }^{3}$ No analizamos los remates cuyo origen es una condición de mora en el pago de la patente por no uso de DAA ya asignados, opción de remate introducida en la reforma del CA de 2005 (Arts. 129 bis11, bis15 y bis16). A fines de 2017 existían más de 3.200 DAA con remates pendientes de realización, fruto de morosidad en el pago de patente por no uso. Trabas y costos burocráticos han entorpecido la realización de estos remates (El Mercurio 2017, B7; El Mercurio 2018b, B16).
} 
Una proporción no despreciable de los remates convocados fueron declarados desiertos $(50 \%$ de los convocados para DAA/NC y $74 \%$ de los convocados para superficies de exploración). En los remates que sí ocurrieron, con frecuencia asistieron pocos participantes (en 2/3 de los remates realizados, asistieron no más de dos participantes). $\mathrm{Y}$ en los remates con pocos participantes, predominaron adjudicaciones a los precios mínimos de oferta.

¿Por qué no se han cumplido las expectativas de los promotores de la reforma del CA de 2005, en términos del objetivo que buscaba dinamizar el uso de remates como mecanismo asignador de nuevos derechos de agua? En este trabajo se analizan distintas hipótesis que ayudan a entender lo ocurrido. Las causas posibles son diversas; entre otras se incluyen: problemas de sobreotorgamiento de derechos de agua en algunas cuencas; participación de agentes especuladores en el proceso de solicitud de nuevos derechos; acuerdos ex ante entre los interesados en participar en un remate (para reducir el costo de adjudicarse el nuevo derecho); y retrasos significativos en el proceso administrativo (en la DGA) que resuelve las solicitudes de nuevos DAA.

La siguiente sección describe las reglas para asignar DAA en Chile, antes y después de la reforma del CA de 2005. Las secciones 3 a 5 describen los datos usados y explican la frecuencia de uso del mecanismo de remate para asignar DAA durante el período analizado. Las secciones 6 y 7 analizan hipótesis y evidencia sobre los hechos descritos. La sección 7 plantea desafíos para futuros análisis más detallados, en relación con (i) el patrón recurrente de muy pocos participantes por remate (en una fracción significativa de los remates ocurridos) y (ii) las consecuencias de decisiones de diseño licitatorio (sobre el mecanismo de licitación y los objetos en venta) que fueron priorizadas en los remates realizados durante el período analizado. La sección 8 presenta conclusiones. Los Anexos 1 y 2 reportan datos complementarios al texto central.

\section{ENTORNO LEGAL Y ADMINISTRATIVO DE LOS DAA}

\subsection{Tipos de DAA}

El CA de 1981 normó los DAA en Chile, estableciendo derechos de aprovechamiento que permiten el uso y goce de aguas superficiales y subterráneas. El agua solicitada es asignada por la DGA. Los DAA pue- 
den ser otorgados en tres categorías: (i) según tipo de uso (consuntivo o no consuntivo), (ii) según disponibilidad de caudal (permanente o eventual) y (iii) según frecuencia de uso (continuo, discontinuo o alternado).

Los derechos no consuntivos (DAA/NC, en adelante) permiten al propietario utilizar el agua otorgada, pero esta debe ser luego restituida al cauce original sin producir alteración negativa, ni en calidad ni en caudal. Así, un caudal de DAA/NC puede ser asignado en un mismo cauce varias veces, en tanto lo permitan las condiciones legales y geográficas para su explotación. Los DAA/NC son utilizados principalmente en generación eléctrica y también, en menor medida, en acuicultura. Los derechos consuntivos (DAA/C, en adelante) permiten a su propietario extraer el caudal otorgado y consumirlo en su totalidad, sin necesidad de restituirlo.

Un DAA permanente indica el flujo de agua máximo que se permite extraer al titular. En el caso de que no hubiese caudal suficiente para satisfacer a todos los DAA permanentes, el titular de cada DAA puede extraer una proporción de los flujos disponibles (según el porcentaje que cada DAA representa en el caudal total otorgado en un cierto cauce). Los derechos eventuales permiten a su titular extraer agua únicamente si se han satisfecho todos los DAA permanentes; son, por lo tanto, derechos residuales.

Los DAA se pueden usar en forma continua, discontinua o alternada. Los DAA de ejercicio continuo permiten usar el agua en forma ininterrumpida; los DAA de ejercicio discontinuo solo permiten usar el agua durante ciertos períodos. En los DAA de ejercicio alternado, el uso del agua se comparte entre dos o más usuarios que se turnan en su uso. Los distintos tipos de DAA se otorgan en función de la solicitud y disponibilidad del recurso, siendo algunos caudales medidos como promedio mensual (aguas superficiales) y otros como caudales-año (aguas subterráneas).

\subsection{Asignación inicial y transferibilidad de DAA}

El CA de 1981 definió las modalidades de asignación inicial (gratuita o vía remate), permitiendo la libre transferibilidad y el libre uso de los DAA, independientemente de cuándo o cómo se otorgaron los derechos. Para evitar daños a terceros, producto de una transferencia de 
DAA, la DGA puede limitar la transferibilidad del DAA en casos en que el intercambio de DAA implique cambio en el punto de captación de las aguas. En estos casos, la transferibilidad requiere autorización previa de la DGA.

La reforma del CA en 2005 (Ley 20.017), sin cambiar la esencia del sistema, modificó aspectos procedimentales asociados al uso del remate como mecanismo de asignación inicial de DAA. A continuación se describe la operatoria del mecanismo de asignación de DAA y los cambios introducidos por la reforma del año 2005.

\section{Asignación inicial de derechos}

Para obtener una asignación inicial de DAA se requiere solicitarla a la DGA. Se otorga lo solicitado si existe disponibilidad para satisfacer el total de caudales demandados, sin afectar derechos de terceros. Al no existir rivalidad por un mismo DAA, el derecho se otorga en forma gratuita. Cuando existen dos o más solicitudes por el mismo caudal y el agua disponible es insuficiente para la totalidad de caudal solicitado, la asignación de DAA se otorga al ganador de un remate al mejor postor (mayor precio), mecanismo que dirime la asignación de DAA superficiales, subterráneas y solicitudes rivales sobre el derecho a explorar una determinada fuente de agua subterránea (si está localizada en terreno fiscal).

\section{Proceso de solicitud de DAA}

Se debe presentar una solicitud que contenga antecedentes que permitan identificar al solicitante, el tipo de derecho demandado y una memoria explicativa que justifique técnicamente el caudal solicitado y el uso manifestado por el solicitante.

La solicitud de DAA abre un expediente para tramitar el respectivo proceso administrativo. Existiendo disponibilidad, la DGA puede otorgar la constitución inicial de un DAA mediante resolución administrativa, sin pago desde el adjudicatario. La DGA realiza estudios hídricos para determinar los caudales disponibles. La periodicidad de estos estudios depende del presupuesto disponible, lo que afecta los plazos en que se desarrolla el proceso de otorgamiento. 
Tabla 1. USO DE REMATE: CAMBIOS INTRODUCIDOS A PARTIR DE LA REFORMA DE 2005

\begin{tabular}{|c|c|c|}
\hline Tema & (1) Código de 1981 & $\begin{array}{l}\text { (2) Desde } 2005 \text { hasta la } \\
\text { actualidad }\end{array}$ \\
\hline $\begin{array}{l}\text { 1) Plazo para publicar la } \\
\text { solicitud al público }\end{array}$ & $\begin{array}{l}15 \text { dias desde presentada } \\
\text { la primera solicitud }\end{array}$ & $\begin{array}{l}30 \text { días desde presentada } \\
\text { la primera solicitud }\end{array}$ \\
\hline $\begin{array}{l}\text { 2) Plazo para que un } \\
\text { tercero presente oposición }\end{array}$ & $\begin{array}{l}30 \text { días desde presentada } \\
\text { la primera solicitud }\end{array}$ & Ídem \\
\hline $\begin{array}{l}\text { 3) Plazo para que un terce- } \\
\text { ro presente otra solicitud }\end{array}$ & $\begin{array}{l}30 \text { días desde presentada } \\
\text { la primera solicitud }\end{array}$ & $\begin{array}{l}6 \text { meses desde presentada } \\
\text { la primera solicitud }\end{array}$ \\
\hline $\begin{array}{l}\text { 4) Aviso de remate en } \\
\text { medio escrito }\end{array}$ & $\begin{array}{l}1 \text { vez en diario de Santiago } \\
\text { y } 1 \text { vez en diario local, } 10 \\
\text { días antes del remate }\end{array}$ & $\begin{array}{l}\text { Ídem, pero agrega no- } \\
\text { tificaciones por carta a } \\
\text { solicitantes }\end{array}$ \\
\hline $\begin{array}{l}\text { 5) Tipo de derechos que } \\
\text { pueden ser rematados }\end{array}$ & Superficiales & $\begin{array}{l}\text { Superficiales y subterrá- } \\
\text { neos }\end{array}$ \\
\hline $\begin{array}{l}\text { 6) ¿Quiénes pueden ser } \\
\text { postores en el remate? }\end{array}$ & $\begin{array}{l}\text { Solo participan los soli- } \\
\text { citantes que originan la } \\
\text { subasta y el Fisco }\end{array}$ & $\begin{array}{l}\text {-En aguas superficiales: } \\
\text { abierto a todo interesado } \\
\text {-En aguas subterráneas: } \\
\text { solo los solicitantes } \\
\text {-El Fisco }\end{array}$ \\
\hline $\begin{array}{l}\text { 7) Caudal a rematar y } \\
\text { división del objeto }\end{array}$ & $\begin{array}{l}\text { Se definen las cuotas según } \\
\text { el mínimo caudal solicitado. } \\
\text { Se remata cada cuota. Se } \\
\text { da preferencia al ganador } \\
\text { de una cuota previa para } \\
\text { adjudicarse el resto de } \\
\text { cuotas hasta completar su } \\
\text { caudal solicitado }\end{array}$ & $\begin{array}{l}\text { Ídem, pero se incorpora } \\
\text { desde } 2008 \text { un tipo de } \\
\text { reglamento denomina- } \\
\text { do "Manual de normas } \\
\text { y procedimientos para } \\
\text { la administración de } \\
\text { recursos hidricos" (Parte } \\
\text { VIII: Remates), en adelante } \\
\text { Manual/2008, que busca } \\
\text { uniformar criterios respec- } \\
\text { to de temas procedimen- } \\
\text { tales }\end{array}$ \\
\hline 8) Bases para el remate & $\begin{array}{l}\text { Son responsabilidad y a } \\
\text { discreción de la DGA }\end{array}$ & $\begin{array}{l}\text { Ídem, pero luego se incor- } \\
\text { poran el Manual/2008 y } \\
\text { un reglamento (Decreto } \\
203 / 2013 \text { ) sobre normas } \\
\text { de exploración y explota- } \\
\text { ción de aguas subterrá- } \\
\text { neas en terrenos fiscales }\end{array}$ \\
\hline $\begin{array}{l}\text { 9) Exploración de aguas } \\
\text { subterráneas }\end{array}$ & $\begin{array}{l}\text { No existe la opción de usar } \\
\text { remate para este tipo de } \\
\text { derecho }\end{array}$ & $\begin{array}{l}\text { Asignación via remate en el } \\
\text { caso de que exista super- } \\
\text { posición de superficie entre } \\
\text { dos o más solicitudes }\end{array}$ \\
\hline
\end{tabular}

Fuente: elaboración propia. 
Podría presentarse más de una solicitud sobre las mismas aguas, debiendo la DGA acreditar la existencia de aguas disponibles para asignar nuevos DAA. Las solicitudes deben ser publicadas en el Diario Oficial y en un periódico local (de la región involucrada en la solicitud); además se debe difundir el extracto de la solicitud en medios radiales del sector individualizado en la solicitud.

A partir de la reforma del CA de 2005, se pueden recibir nuevas solicitudes de DAA dentro de un plazo de seis meses, contados desde la primera solicitud (para una comparación pre- versus posreforma de 2005, ver Tabla 1). Finalizado este plazo, resueltas todas las oposiciones y en caso de no acreditarse la existencia de caudal suficiente para satisfacer todas las solicitudes presentadas, surge una situación de competencia entre dos o más solicitantes, la que se debe resolver vía remate.

El mecanismo del remate ha estado presente desde el CA de 1981, pero la reforma del año 2005 introdujo un conjunto de cambios procedimentales relacionados con la opción de utilizar el mecanismo de remate. Entre otros cambios, a partir de 2005 se permite que concurran al remate agentes distintos de los solicitantes originales (en el caso de aguas superficiales). El resto de las normas sobre el funcionamiento de los remates se mantuvo según lo definido en el CA de 1981. El tipo de remate corresponde a un remate (oral) al mejor postor (precio más alto). Otros detalles sobre la forma de operación del remate se definen en las bases que la DGA debe publicar previo a cada remate.

\subsection{Sobre las reglas del remate}

a) Objeto(s) por rematarse: La DGA determina la división en cuotas del caudal total solicitado, siendo las cuotas los objetos por rematar. Las cuotas se pueden determinar según dos criterios básicos: (i) el menor caudal solicitado (Art. 145, CA) o (ii) el máximo común divisor entre todos los caudales solicitados (cap. 8, Manual/2008). Con estos criterios se busca que las cuotas definidas puedan satisfacer las solicitudes de menor caudal; en la práctica, el criterio predominante ha sido (ii). Las cuotas suelen ser heterogéneas, pudiendo incluir caudales de distintos tipo, disponibilidad y frecuencia de uso.

La DGA determina qué combinaciones de caudal (tipo de DAA y volumen) definirán las cuotas por rematar, buscando satisfacer los cau- 
dales solicitados, dada el agua disponible. La definición de las cuotas es específica a cada remate. Cada cuota se expresa según su respectivo 'caudal medio mensual', una métrica promedio-mes sobre la magnitud del caudal otorgado en cada cuota. Definidas las cuotas, la DGA especifica en las bases del remate el orden de venta de las cuotas.

b) Tipo de remate: Es de tipo oral y "el derecho de aprovechamiento por cada unidad [i.e., cuota] se adjudicará al mejor postor [precio más alto] y así sucesivamente hasta que se termine el total del caudal ofrecido" (Art. 145, CA). Además, el ganador de la primera cuota rematada, que a la vez sea uno de los solicitantes originales del DAA disputado, tiene el derecho preferente a adquirir, al mismo precio de la primera cuota adjudicada, las cuotas adicionales que pueda requerir hasta completar todo su caudal solicitado.

En lo que sigue se describe el proceso administrativo que debe seguir, posreforma de 2005, una solicitud de DAA para finalizar en la asignación del derecho solicitado. Existen tres etapas.

\section{Etapa 1: Solicitudes y plazos}

Iniciado el proceso de solicitud por un nuevo DAA, se puede presentar una oposición a esa solicitud dentro de los primeros treinta días (desde el inicio de la solicitud), por terceros que consideren afectado un derecho preexistente. Tal oposición podría terminar en la denegación de la solicitud. Además, dentro de un plazo de seis meses, desde el ingreso de la primera solicitud, se podría presentar a la DGA otra solicitud 'sobre las mismas aguas' o 'superficies de exploración', lo que gatillaría una condición de competencia por el otorgamiento de esos derechos. Expirado este plazo, la DGA debe iniciar los estudios de disponibilidad de caudal. Si existe suficiente disponibilidad de agua para satisfacer todas las solicitudes, la asignación es gratuita. De lo contrario, se debe convocar a remate.

\section{Etapa 2: Estudio y bases de remate}

La no acreditación (por parte de la DGA) de caudal total disponible (denótese este por $Q_{D G A}$ ) implica que no se puede otorgar la totalidad de los caudales solicitados. Pero la disponibilidad acreditada puede ser 
mayor que alguna de las solicitudes individuales; en este caso se gatilla el uso del remate. La DGA define las bases del remate, una vez que se han resuelto todas las oposiciones y la determinación de $Q_{D G A}$. En la práctica, el proceso de estudio y resolución final puede prolongarse por varios años. ${ }^{4}$

Aparte de la definición de bases específicas a cada remate, otras reglas, necesarias para desarrollar los remates de DAA, se encuentran definidas en el Manual/2008. El capítulo 8 del Manual corresponde a un reglamento de facto para efectuar remates para asignar DAA. El Manual/2008 define: (i) la forma en que se deben determinar las cuotas por rematar, en función del $Q_{D G A}$ acreditado y del caudal mínimo contenido en las solicitudes; y (ii) el cálculo del precio mínimo de adjudicación al rematar distintos tipos de DAA y derechos de exploración de aguas subterráneas. A continuación, se resumen los criterios atendidos por el Manual/2008 para definir parte de las variables consideradas.

1) Caudal total disponible $\left(Q_{D G A}\right)$ : Se determina mediante un estudio técnico de la DGA. Contiene una estimación del caudal medio mensual para uso consuntivo y no consuntivo, considerando los tipos de caudal solicitados (permanente, eventual, continuo, discontinuo o alternado). Este estudio se usa para dictar las bases del remate.

2) Valor comercial de referencia para los DAA por rematarse: La DGA determina para cada cuota por rematar: (i) un valor comercial de referencia $(V C)$ y (ii) un precio mínimo de postura, calculado en función de $V C$.

En el caso de DAA/C (para aguas subterráneas y superficiales), $V C$ se determina sobre la base de precios en el mercado secundario de DAA. En el caso de DAA/NC utilizados para generación eléctrica, $V C$ se calcula a partir de un estimado de la potencia eléctrica generable en el punto de restitución definido en la solicitud; potencia que es función de: (a) el desnivel geográfico determinado por la diferencia entre la cota del punto de captación y la del punto de restitución, y (b) la estimación

\footnotetext{
${ }^{4}$ Por ejemplo: el proceso del remate de Río Manso (DAA/NC, Tabla A1.3, remate \#7) demoró cerca de diez años en completarse, mientras que el proceso para el remate sobre el río Carilafquén (DAA mixtos, Tabla A2.3, remate \#8) tomó tres años en total. El inciso segundo del Art. 134 (CA) define cuatro meses como plazo legal máximo para emitir el informe y resolución de la DGA. Pero en la práctica este plazo no es exigible, en tanto la DGA no cuente con los informes técnicos sobre $Q_{D G A}$.
} 
del caudal medio anualizado. ${ }^{5}$ En este caso, la DGA estima el $V C$ por cuota según:

$$
V C_{c}=\left(P_{c} \cdot \text { Precio }_{\text {Nudo }}^{\text {Ref }}\right)
$$

en donde Precio $_{\text {Nudo }}^{\text {Ref }}=$ Precio Nudo de referencia en el mercado de generación eléctrica. En la práctica, la DGA ha utilizado un valor fijo, constante a través del tiempo, para valorizar Precio $_{N u d o}^{R e f}$. El valor usado por la DGA aparece en el Manual/2008.

En el caso de DAA/NC para uso en piscicultura, para determinar $V C$ la DGA ha utilizado otro valor fijo (constante en el tiempo), también mencionado en el Manual/2008. Por lo tanto, en el caso de los derechos DAA/NC, las estimaciones del valor $V C$ por cuota no se basan en valores de mercado, sino en valores fijos predeterminados.

3) Precio mínimo de postura $\left(P^{M i n}\right)$ : La DGA lo calcula según: ${ }^{6}$ $P^{\text {Min }}=(\mathrm{VC} \cdot 0,1)$. Por lo tanto, los $P^{\text {Min }}$ considerados al rematar cuotas de $\mathrm{DAA} / \mathrm{NC}$ tampoco guardan relación con precios de mercado de los derechos en oferta. Los DAA/NC representan en torno a 3/4 del total de caudales rematados durante 2005-2018. Por otra parte, $P^{M i n}$ se hace público con las bases del remate, lo que suele ocurrir con hasta dos meses de antelación a la realización del remate. ${ }^{7}$

4) Caudales eventuales versus permanentes: La DGA ha establecido que los caudales eventuales corresponden a un tercio de un equivalente caudal permanente; esto, para definir los caudales totales (cuotas) por rematar y sus respectivos $P^{\text {Min }}$.

5) Las bases de cada remate definen la garantía de participación, ${ }^{8}$ cauciones, sanciones, plazo de inscripción de dominio de los DAA por adjudicarse, entre otros.

\footnotetext{
${ }^{5}$ La DGA calcula el potencial de generación a partir de: $P_{c}=\left(0,0098 \cdot Q_{\text {medio,c }} \cdot H_{c}\right)$, en donde: $P_{c}=$ Potencia equivalente (en $\left.\mathrm{MW}\right)$, una medida de capacidad de generación eléctrica, para la cuota c; $Q_{\text {medio }}=$ Caudal medio anualizado; y $H=$ Desnivel entre el punto de captación y restitución; siendo $Q_{\text {medio }}$ y $H$ valores específicos a la cuota c.

${ }^{6}$ Ver numeral 8.3.2 del Manual/2008. En las entrevistas con personal de la DGA no se obtuvo ninguna explicación que permitiera justificar el uso del coeficiente 0.1 .

${ }^{7}$ Esto puede facilitar acuerdos de coordinación ex ante entre los interesados en participar en un remate, para disminuir el valor de los precios de adjudicación.

${ }^{8}$ La garantía exigida para participar en un remate de DAA, y también para áreas de exploración de aguas subterráneas, equivale al 10\% del precio mínimo del total de cuotas por rematarse.
} 


\section{Etapa 3: Ejecución del remate}

Las ofertas se realizan a viva voz, hasta lograr un precio máximo por cuota. No existe regla que regule la modalidad de desarrollo de las ofertas de precio (en cuanto a sus incrementos o intervalos de tiempo).

A partir de la reforma de 2005, la DGA debe levantar un acta con los resultados de cada remate. Las actas son públicas. Pero a la fecha de nuestra investigación, no todas las actas estaban disponibles en el sitio web de la DGA. Tuvimos acceso a actas (con información detallada sobre cada remate convocado) en el $93 \%$ del total de los remates convocados. Respecto de los remates convocados que no tenían un acta disponible (nueve en total), todos ellos fueron remates declarados desiertos. En estos casos dispusimos de información muy general (número de remates convocados y declarados desiertos, según tipo de derecho solicitado, y número de solicitantes de nuevos derechos, por remate convocado).

El adjudicatario de cada cuota rematada puede pagar el precio de adjudicación en diez anualidades. No obstante, no existe normativa clara, ni en el CA ni en las bases de los remates analizados, que estipule la caducidad del derecho adquirido vía remate, en caso de incumplir los pagos convenidos, después de pagada la primera cuota. ${ }^{9} \mathrm{Y}$ esto, a pesar de que el CA (Art. 143) señala que "Las bases [de los remates] establecerán, también, las sanciones por incumplimiento de las condiciones específicas que se exijan a los adjudicatarios". Al respecto, existe un plazo máximo de treinta días (desde la fecha de la resolución que asigna el DAA obtenido vía remate) para inscribir el DAA adjudicado en el respectivo Conservador de Bienes Raíces (CBR) y pagar la primera cuota a la DGA, previo a lo cual el adjudicatario debe reducir a escritura pública el DAA adquirido. En la práctica surgen problemas que hacen engorrosa la notificación de situaciones de morosidad por cuotas impagas de DAA y su posterior llamado a nuevo remate. ${ }^{10}$ Añade com-

\footnotetext{
${ }^{9}$ Según información obtenida en la DGA (Francisco Puelma, staff de la DGA), a la fecha no existen antecedentes sobre la imposición de caducidad por parte de la DGA, de DAA adjudicados vía remate y respecto de los cuales, posterior al pago de la primera cuota, se hubiese producido un incumplimiento en los restantes pagos.

${ }^{10} \mathrm{Si}$ se produce morosidad, la Tesorería debe notificar al moroso y luego publicar que se llevará a cabo un nuevo remate. Pero la notificación a menudo falla, pues existe la práctica de transferir el DAA cuando se aproxima la fecha de notificación por morosidad. Esto obliga a reiniciar el proceso de cobranza. Además, para notificar la morosidad de pagos
} 
plicación el hecho de que muchos poseedores de DAA no los inscriben en los respectivos CBR, para evitar el cobro de la patente por no uso, lo que imposibilita iniciar el proceso de cobranza judicial (El Mercurio 2018a). En la práctica, no ha existido normativa clara ni institucionalidad eficaz para aplicar sanciones por morosidad en el pago de cuotas del precio de adjudicación de DAA vía remates. ${ }^{11}$

\section{USO DEL MECANISMO DEL REMATE}

Desde la vigencia del CA de 1981 y hasta su modificación en 2005, solo se habrían realizado cuatro remates para asignar DAA. ${ }^{12}$ Previo a la reforma de 2005, no existen actas públicas sobre los remates realizados; por lo tanto, no hay datos específicos al respecto. Información (solo

asociados a la tenencia de DAA (por ejemplo, por morosidad en la patente por no uso) y luego iniciar el proceso de cobranza judicial, la Tesorería debe incurrir en varios gastos que luego generan ingresos que, en gran medida, van a fondos comunales y regionales. Así, el organismo encargado de cobrar tiene pocos incentivos para destinar sus recursos a este fin (El Mercurio 2018a, A3; El Mercurio 2018c, A3).

${ }^{11}$ En la inscripción del DAA en el respectivo CBR, se debe consignar hipoteca a favor fiscal en tanto el adjudicatario del DAA mantenga deuda vigente con el Fisco. Si bien la DGA sería la institución mandatada para recibir los pagos y luego ponerlos a disposición de la Tesorería General de la República (ver Comisión Especial Mixta de Presupuesto del Senado 2016), Comisión Especial Mixta de Presupuesto del Senado; octubre 19 de 2016), mediante entrevistas con personal de la DGA no pudimos constatar que, a la fecha, la DGA haya iniciado juicios de cobro respecto de obligaciones impagas, que sí han existido, de deudores adjudicatarios de DAA vía remate.

12 Existe muy escasa información al respecto. La mención pública más notoria fue realizada en 2007 por un ex director de la DGA, Rodrigo Weisner. En entrevista sobre los cambios introducidos el año 2005 al CA, Weisner señalaba: "el mecanismo del remate casi nunca se dio, ya que los oferentes se solían poner de acuerdo. Imagínate que entre el año 1981 y 2005 hubo sólo dos remates" (destacado nuestro). Weisner (2007) además aseveró: "Hoy el mecanismo de remate funciona a las mil maravillas y es el que prima para hacerse de un derecho de agua, ya que la mayoría están inscritos". Asimismo, durante la discusión parlamentaria para la redacción de la Ley 20.017 (que dio lugar al actual CA de 2005), el ministro de Obras Públicas de la época (Ricardo Lagos) aseveró que [previo a la reforma del CA en 2005] "sólo habrían ocurrido un par de remates... y no ha habido un mayor número, simplemente porque no se han producido más situaciones, lo cual es una buena demostración de lo que estamos diciendo: que el Código no está funcionando en este aspecto. Ojalá hubiera habido más remates, pero no ha existido posibilidad" (Lagos 2007; destacado nuestro). 
general) sobre los remates realizados antes del año 2005 se obtuvo en artículos de prensa y en la historia de la ley sobre la reforma de $2005 .{ }^{13}$

Una de las principales hipótesis (prereforma de 2005) sobre por qué el mecanismo de remate se usó tan esporádicamente antes del año 2005, planteaba que la ley contemplaba un plazo 'demasiado breve' para realizar una segunda solicitud, competitiva con una ya existente; de esta forma se dificultaba el poder configurar uno de los requisitos básicos para usar el mecanismo del remate para asignar DAA (Congreso Nacional 2005). Hasta la reforma de 2005, la ley estipulaba un plazo máximo de treinta días, desde la fecha de ingreso de la primera solicitud, para ingresar nuevas solicitudes u oposiciones sobre un mismo caudal (Tabla 1). ${ }^{14}$

En la reforma de 2005 se incorporaron cambios legales para facilitar la realización de remates al requerirse dirimir entre solicitudes rivales de DAA (Congreso Nacional 2005, 1241-244). Los principales cambios introducidos fueron (ver Tabla 1): (1) aumentar el plazo para presentar solicitudes rivales; (2) agregar la opción de usar remates para también asignar aguas subterráneas y superficies de exploración de aguas subterráneas; y (3) permitir la participación de cualquier postor interesado en remates de aguas superficiales (previo al CA de 2005 solo se permitía participar a los solicitantes directos del DAA). Relacionado a (3), la Resolución 1800 de la DGA (julio de 2010) precisó que se anu-

\footnotetext{
${ }^{13}$ La primera instancia de remates correspondió a tres remates para asignar caudales en la cuenca del río Maipo (RM), en septiembre y octubre de 1981, luego de la promulgación del CA del 13 de agosto de 1981. En esos remates se adjudicaron DAA a las ex empresas sanitarias EMOS, Lo Castillo y a la (ex) Minera Disputada de Las Condes. La segunda instancia corresponde a un remate realizado en la cuenca del Río Correntoso (Provincia de Llanquihue, X Región), involucrando un pequeño caudal (5 1/s). A este remate solo concurrió un solicitante y el correspondiente DAA se adjudicó al precio mínimo.

${ }^{14}$ Una pregunta que surge es por qué los legisladores habrían estipulado un plazo así de breve en el texto legal del CA de 1981. Cuesta imaginar que los legisladores no pudieran prever las complicaciones prácticas que este plazo podía crear. Cabe pensar en una hipótesis de captura regulatoria del voto mayoritario en el Poder Legislativo. Pero no tenemos evidencia para testear esta conjetura. Bauer $(1997,649)$ plantea una hipótesis alternativa: "Later the military government intervened directly to prevent auctions in several cases with intersectoral implications, particularly involving hydroelectric projects. The government was unwilling to leave such crucial allocative decisions to the free market, and either denied the applications or simply assigned the rights to the applicant preferred on the basis of 'exceptional circumstances and general interest' (based on Art. 148 of the Water Code)".
} 
la el llamado a remate si ocurre desistimiento de un solicitante, previo a la dictación de las bases del remate, en un total de dos titulares de solicitudes ingresadas.

\section{DATOS USADOS}

La información sobre los remates analizados proviene de datos publicados (actas) en el sitio web de la DGA. Esta información luego se registra en el Catastro Público de Agua. ${ }^{15}$ Las actas disponibles también incluyen casos de remates declarados desiertos. ${ }^{16}$ A partir de las actas se generó una base de datos que describe cada remate según: cantidad y tipos de caudales (cuotas) ofertados (tipo de uso, frecuencia y disponibilidad); y precios mínimos de oferta y de adjudicación.

\section{Caudal equivalente y precios unitarios de adjudicación}

Para determinar el caudal disponible a rematar, la DGA estima caudales promedio mensual, en términos anualizados, los que se pueden rematar en una o varias cuotas. Las cuotas pueden incluir distintos tipos de DAA (permanentes, continuos, eventuales o discontinuos), pudiendo existir cuotas con caudales no directamente comparables. Para resolver este problema, nuestro análisis utiliza una unidad de caudal comparable: Caudal Equivalente, expresado como un promedio mensual (anualizado) y que corresponde a un flujo permanente de DAA.

Para determinar el Caudal Equivalente por cuota, se transforman los correspondientes caudales eventuales y discontinuos a sus equivalentes permanentes y continuos. Se utiliza la regla usada por la DGA (caudales eventuales o discontinuos corresponden a un tercio de sus equivalentes caudales permanentes o continuos).

Obtenidos los caudales equivalentes se calculan los correspondientes precios unitarios de adjudicación ( $P^{a}$, expresado en $\left.\$ / 1 / \mathrm{s}\right)$, por cuota

\footnotetext{
${ }^{15}$ El sitio web de la DGA cuenta con registros detallados, aunque no completos, de DAA constituidos por región, sujetos a actualización periódica. El Catastro Público de Agua está disponible en el sitio web de la DGA. Este catastro contiene información histórica (aunque no $100 \%$ regularizada) sobre el otorgamiento de DAA en Chile. Nuestro estudio lo ha usado para comparar, durante el período analizado, las asignaciones de DAA realizadas vía remate con aquellas otorgadas mediante el régimen general.

${ }^{16}$ Remates convocados, pero en los cuales no se presentaron ofertas.
} 
rematada. Luego se calculan precios unitarios promedio de adjudicación por remate; en cada remate, los precios unitarios de adjudicación de las distintas cuotas asignadas se promedian entre sí, ponderando cada precio por la proporción de caudal vendido por cuota, respecto del caudal total adjudicado en ese remate.

\section{SOBRE LOS REMATES OCURRIDOS DE DAA}

Entre junio de 2005 y diciembre de 2018, el número total de remates convocados fue de 134, incluyendo remates para asignar nuevos DAA (aguas superficiales y subterráneas) y derechos para exploración de aguas subterráneas. ${ }^{17}$ Este total incluye todos los remates convocados (con y sin acta publicada en el sitio web de la DGA).

La Tabla 2 describe el número total de los remates convocados a nivel nacional para asignar nuevos derechos de agua, incluyendo los remates declarados desiertos y aquellos realizados. La columna (4) reporta el caudal total asignado en los remates realizados, según tipo de DAA. La primera fila (DAA/C) incluye remates de aguas consuntivas (superficiales y subterráneas). La segunda (DAA/NC) reporta el número de remates de DAA de aguas solo NC. La categoría de DAA 'mixtos' reporta remates en los que se ofertaron derechos tipo $\mathrm{C}$ y NC, pero sus actas no detallan cuáles cuotas corresponden a derechos tipos $\mathrm{C}$ y cuáles a NC. La cuarta fila reporta remates de superficies de exploración de aguas subterráneas.

En términos de caudales de DAA asignados durante 2005-2018, incluyendo DAA/C y DAA/NC, el total de caudales asignados vía remate ( $319 \mathrm{~m}^{3} / \mathrm{s}$; filas 1 a 3 en Tabla 2 ) equivale solo al $0,8 \%$ de los caudales totales asignados a nivel nacional mediante el régimen general (asignación gratuita, i.e. $\left.41.408 \mathrm{~m}^{3} / \mathrm{s}\right),{ }^{18}$ incluyendo todos los tipos de DAA asignados durante junio de 2005 y hasta fines de diciembre de 2018. Así, los caudales rematados representan una proporción muy marginal en relación al total de caudales asignados de forma gratuita. ${ }^{19}$

\footnotetext{
${ }^{17}$ Las aguas subterráneas se miden en litros/segundo/año (1/s/año), mientras que los caudales de DAA/NC en $\mathrm{m}^{3} / \mathrm{s}$ y los caudales consuntivos superficiales en $1 / \mathrm{s}$.

${ }^{18}$ De este total, $39.961 \mathrm{~m}^{3} / \mathrm{s}(96,5 \%)$ correspondieron a DAA/NC y $1.447 \mathrm{~m}^{3} / \mathrm{s}(3,5 \%)$ a DAA/C (ver Tabla 4, más adelante).

${ }^{19}$ La información sobre asignaciones de DAA vía régimen general (asignación gratuita) se obtuvo del Catastro Público de Aguas. Para este cálculo se incluyeron todos los tipos de caudales asignados e inscritos en los CBR.
} 
Tabla 2. NÚMERO TOTAL DE REMATES A NIVEL NACIONAL (JUNIO DE 2005 A DICIEMBRE DE 2018)

\begin{tabular}{lcccc}
\hline Tipo de DAA rematado & $\begin{array}{c}(1) \\
\text { Convocados }\end{array}$ & $\begin{array}{c}(2) \\
\text { Desiertos }\end{array}$ & $\begin{array}{c}(3) \\
\text { Realizados }\end{array}$ & $\begin{array}{c}(4) \\
\text { Caudales } \\
\text { (o superficies) } \\
\text { totales asignados }\end{array}$ \\
\hline 1) DAA/C & 11 & 1 & 10 & $5,75\left(\mathrm{~m}^{3} / \mathrm{s}\right)$ \\
2) DAA/NC & 54 & 27 & 27 & $231,1\left(\mathrm{~m}^{3} / \mathrm{s}\right)$ \\
3) Remates (DAA) 'mixtos' & 19 & 11 & 8 & $82,1\left(\mathrm{~m}^{3} / \mathrm{s}\right)$ \\
4) Superficies de & 50 & 37 & 13 & $70.433(\mathrm{ha})$ \\
exploración & 134 & 76 & 58 & \\
Total & & & & \\
\hline
\end{tabular}

Fuente: elaboración propia sobre la base de datos obtenidos en www.dga.cl.

El total de remates convocados de DAA (aguas superficiales y subterráneas) alcanza a 84 (filas 1 a 3), de los cuales 45 se realizaron. Respecto de los remates declarados desiertos, su incidencia es especialmente alta en las convocatorias para remates de derechos de exploración de aguas subterráneas $(74 \%$ del total de remates convocados en este tipo de derecho); le sigue el caso de remates 'mixtos' de DAA (58\% del total de convocados en esta categoría) y luego los remates de DAA/NC (50\% del total de convocados), mientras que en el caso de DAA/C solo uno de los remates convocados se declara desierto. Más adelante sugerimos hipótesis para explicar este patrón.

La Figura 1 reporta la frecuencia anual de los remates convocados durante 2005-2018. En los primeros años posteriores a la reforma del año 2005 se observa un patrón de leve aumento tanto en el número de remates convocados como en los realizados. El máximo de esta tendencia se alcanza el año 2009; luego ocurre un patrón de disminución, en particular en el número de remates realizados. En el período reportado, destaca la relevancia del número de remates convocados que luego se declaran desiertos (la sección 6 analiza este hecho).

Si bien el patrón inicial de aumento en el número de remates convocados y realizados coincide con los primeros años del período posreforma de 2005, no se puede inferir que tal tendencia haya sido necesaria o únicamente causada por la reforma del año 2005. En el mismo perío- 
Figura 1. NÚMERO DE REMATES CONVOCADOS, POR AÑO (DESDE JUNIO DE 2005 HASTA FINES DE DICIEMBRE DE 2018)

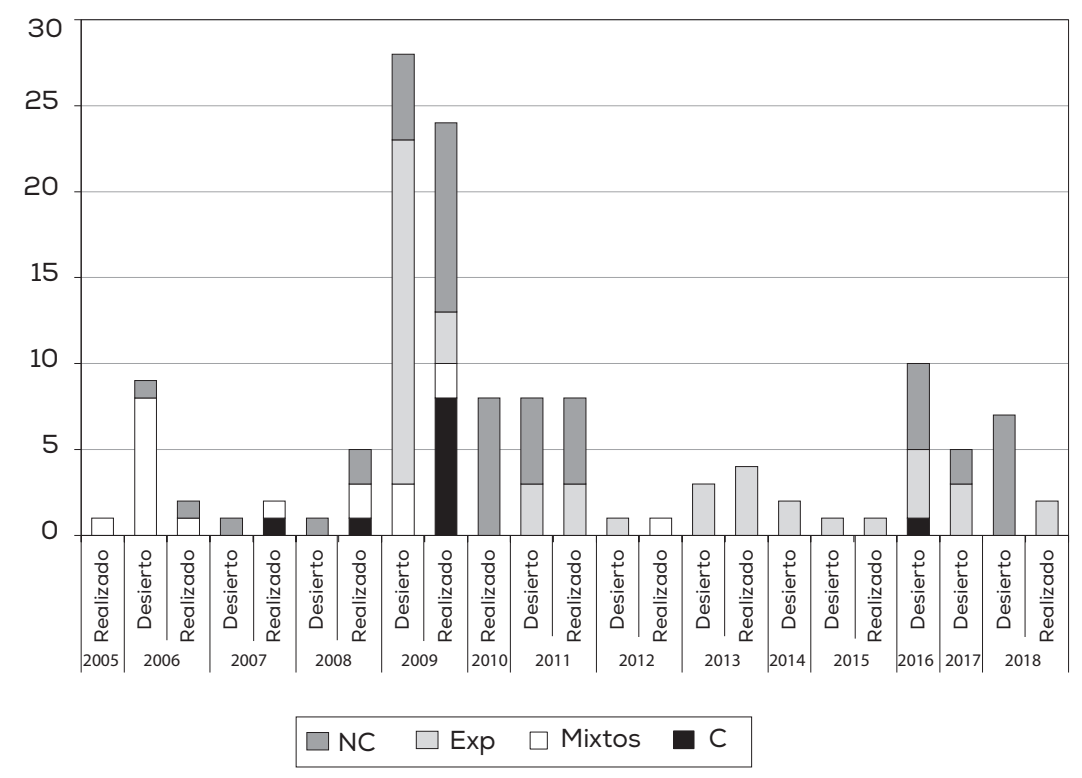

Nota: Incluye remates declarados desiertos. Notación: $\mathrm{C}=$ Consuntivos; $\mathrm{C} / \mathrm{NC}=$ Mixtos; EXP = de Exploración; NC = No Consuntivos.

Fuente: elaboración propia sobre la base de datos obtenidos en www.dģa.cl.

do ocurrieron otros cambios legales que muy probablemente incentivaron un aumento en la demanda por nuevos DAA/NC.

La entrada en vigencia de la Ley $N^{\circ} 20.257$ (en abril de 2008) introdujo la obligación, a partir de enero de 2010, para generadoras con capacidad instalada superior a $200 \mathrm{MW}$, de comercializar (en régimen) ${ }^{20}$ un $10 \%$ de su energía a partir de fuentes de generación renovable no convencional, lo que incluye mini o pequeñas centrales hidroeléctricas de pasada (con potencia de hasta $20 \mathrm{MW}$ ). Como resultado, junto con otros incentivos entonces en boga para invertir en tecnologías 'verdes' de generación eléctrica, ${ }^{21}$ se crearon incentivos para invertir en pequeñas centrales de

\footnotetext{
${ }^{20}$ Esta ley contempló una fase de implementación gradual para esta obligación, partiendo con un 5\% obligatorio durante 2010-2014 hasta alcanzar el 10\% para el año 2024 .

${ }^{21}$ Durante la época analizada también se produjo en Chile un aumento (transitorio) en los incentivos monetarios para desarrollar proyectos de generación de 'energía verde' (Dardati y De Elejalde 2015).
} 
pasada, aumentando la demanda por nuevos DAA/NC. No obstante, luego de una expansión transitoria en estos proyectos, su demanda cayó fruto de un ciclo a la baja en los precios de venta (precio nudo) en generación eléctrica. La caída en la demanda por nuevos DDA/NC se explica además por crecientes dificultades para desarrollar este tipo de proyectos, fruto de conflictos territoriales/sociales (por ejemplo, con comunidades mapuche) y controversias ambientales.

Por lo tanto, no es posible argumentar que el aumento observado en el número de remates de $\mathrm{DAA} / \mathrm{NC}$, que fueron los $\mathrm{DAA}$ rematados con aumentos más relevantes (en términos de los caudales asignados) durante el período analizado, se haya debido únicamente a los cambios introducidos por la reforma del CA del año 2005.

En los anexos se reporta información a nivel de cada uno de los remates ocurridos durante el período analizado. El Anexo 1 reporta información sobre los remates de DAA/ $\mathrm{NC}$, que agrupan el $72,4 \%{ }^{22} \mathrm{del}$ total de los caudales rematados entre 2005 y 2018. El Anexo 2 reporta información sobre los remates de otros tipos de DAA, incluyendo derechos de exploración de aguas subterráneas. En el caso de los DAA/C (caudales superficiales y subterráneos) asignados vía remate, ellos representan solo el 1,8\% del total de los caudales de DAA rematados durante 2005-2018.

\section{6. ¿POR QUÉ OCURRIERON TAN POCOS REMATES DE DAA?}

Consideremos dos opciones generales: (A) que durante el período analizado hayan ocurrido 'pocas' situaciones con solicitudes rivales por un mismo DAA; o (B) habiéndose producido una rivalidad inicial entre solicitudes, con posterioridad uno de dos solicitantes haya desistido de su solicitud y con ello se abortase el llamado a remate. Analicemos primero la opción (B).

Fruto de la situación (B), el aborto de convocatorias a remate pudo haber ocurrido a partir de la vigencia de la Resolución 1800 (julio de 2010). Con anterioridad, el desistimiento de uno de dos solicitantes no detenía el llamado a remate. En este caso, el desistimiento de uno de dos

22 Por falta de información en las actas respectivas, este porcentaje no considera los DAA/NC que pudieron haber sido asignados en remates 'mixtos' (Tabla 1). 
solicitantes ayudaría a explicar la existencia de remates declarados desiertos. Pero ¿por qué un solicitante de DAA podría desistir de su solicitud?

Aparte de la posibilidad de un error inicial de cálculo (dado que perseverar con la solicitud de un nuevo DAA exige incurrir en costos), podría accederse a nueva información que reduce la rentabilidad esperada de perseverar con la solicitud. Una tercera opción es que el conocimiento ex ante de los $P^{\text {Min }}$ considerados al rematar los DAA, reforzado por el hecho de que el valor de los $P^{\text {Min }}$ no necesariamente guarda relación con valores de mercado de los derechos en oferta (ver sección 2.3), podría facilitar acuerdos de coordinación ex ante entre los interesados en participar en un determinado remate, para así disminuir los precios de adjudicación a pagar.

Una cuarta posibilidad es que el desistimiento fuese parte de una estrategia para obtener una renta producto de tal acción. Aunque no disponemos de información que ayude a discriminar directamente entre la validez de estas cuatro alternativas, sí disponemos de alguna evidencia sobre la posible relevancia de la cuarta opción. A continuación, analizamos esta alternativa.

\section{¿Especuladores en el proceso de solicitudes por nuevos DAA?}

La Tabla 3 resume evidencia sobre la ocurrencia de remates declarados desiertos. Esto podría reflejar, al menos en parte, el accionar de agentes especuladores en el proceso de solicitudes por nuevos DAA.

El objetivo de un especulador sería obtener una renta a partir del mero acto de presentar una solicitud rival por un DAA ya solicitado por otro agente, este último con genuino interés en el DAA solicitado. El especulador buscaría obtener un pago desde el solicitante inicial, a cambio de no participar o no competir en el remate. En caso de no existir otros solicitantes del mismo DAA, el retiro ex ante del especulador aseguraría al primer solicitante la obtención del DAA al precio del pago entregado al especulador (pos-Resolución 1800); o si el remate ocurriese (pre-Resolución 1800), en el peor caso $^{23}$ el solicitante ini-

\footnotetext{
${ }^{23}$ La otra opción es que, desistido el especulador, el solicitante inicial tampoco concurra al remate y este se declare desierto. En este caso, el solicitante inicial podría obtener $a$ posteriori el DAA demandado bajo el régimen general de acceso. Pero correría el riesgo de que otro interesado concurra al remate y así obtenga el DAA en cuestión.
} 
Tabla 3. NÚMERO DE REMATES DECLARADOS DESIERTOS (2005-2018)

\begin{tabular}{lcccccccccccccccc}
\hline $\begin{array}{l}\text { Año de } \\
\text { remate }\end{array}$ & 2006 & 2007 & 2008 & 2009 & 2010 & 2011 & 2012 & 2013 & 2014 & 2015 & 2016 & 2017 & 2018 & Total \\
\hline NC & 1 & 1 & 1 & 5 & 0 & 5 & 0 & 0 & 0 & 0 & 5 & 2 & 7 & 27 \\
C & 0 & 0 & 0 & 0 & 0 & 0 & 0 & 0 & 0 & 0 & 1 & 0 & 0 & 1 \\
Mixtos & 8 & 0 & 0 & 3 & 0 & 0 & 0 & 0 & 0 & 0 & 0 & 0 & 0 & 11 \\
$\begin{array}{l}\text { Superficies } \\
\text { de }\end{array}$ & 0 & 0 & 0 & 20 & 0 & 3 & 1 & 3 & 2 & 1 & 4 & 3 & 0 & 37 \\
exploración & & & & & & & & & & & & & & & &
\end{tabular}

Nota: en cada uno de los remates declarados desiertos, el número de solicitantes fue siempre igual a dos.

Fuente: elaboración propia sobre la base de datos obtenidos en www.dga.cl.

cial obtendría el DAA pagando $P^{\text {Min }}$ más el pago al especulador por su desistimiento. El solicitante inicial estaría dispuesto a pagar esta compensación para evitar (a) una prolongación aún más costosa del procedimiento de otorgamiento del DAA solicitado, ${ }^{24}$ o bien, (b) el riesgo de pagar un precio aún mayor para obtener el DAA, en el caso de que el remate ocurra y se produzca rivalidad entre los participantes.

Sería de esperar que el poder explicativo de esta hipótesis aumente, ceteris paribus, a medida que (i) menor sea el costo de presentar una solicitud rival ante la existencia de otra solicitud inicial por el mismo DAA, (ii) más alto sea el valor de mercado del DAA solicitado, o (iii) menor sea el número de otras solicitudes por el mismo DAA. Las tasas de ocurrencia de remates declarados desiertos que se reportan en la Tabla 2 son consistentes con esta hipótesis. Por ejemplo, en cada uno de los remates que se declaran desiertos, el número de solicitantes es siempre igual a dos (Tabla 3), lo que es consistente con la premisa (iii) previa.

En el caso de los DAA/C, si bien se convocaron pocos remates (11 en total), solo uno se declaró desierto (Tabla 2). En el caso de derechos consuntivos, su valor de mercado en general es menor que el valor de

\footnotetext{
${ }^{24}$ El desarrollo administrativo del proceso para rematar un DAA no tiene un plazo perentorio de término para el accionar de la DGA. Así, para garantizar mayor rapidez o seguridad en la obtención del DAA solicitado, en casos con dos solicitantes, el solicitante inicial podría estar dispuesto a pagar para lograr el desistimiento de la solicitud de un agente especulador.
} 
aguas NC. Por lo tanto, la premisa (ii) previa sugiere que, ceteris paribus, la ocurrencia de remates declarados desiertos, producto de la acción de un agente especulador, debiera ser más frecuente en el caso de aguas NC. De hecho, en este último caso la tasa de remates declarados desiertos es del $50 \%$ (respecto del total de los convocados para este tipo de DAA).

Por otro lado, en el caso específico de DAA/C para aguas subterráneas, solicitar este tipo de derechos exige realizar inversiones ex ante no despreciables para 'alumbrar' 25 la fuente de agua solicitada, con lo cual la premisa (i), previa, también tiende a perder validez, de forma similar como ocurre (en términos relativos a los $\mathrm{DAA} / \mathrm{NC}$ ) con la validez de la premisa (ii). Además, las inversiones requeridas por este tipo de solicitudes corresponden mayoritariamente a costos no recuperables (hundidos). Así, el primer solicitante podría disponer de una ventaja competitiva (de 'primer movimiento') por sobre un posterior solicitante/especulador. Esta ventaja, en tanto implique un desincentivo a presentar solicitudes posteriores por un mismo DAA, ayudaría a explicar la muy escasa ocurrencia de remates (solo dos en el período analizado) para asignar DAA/C para aguas subterráneas (Tabla A2.1, Anexo 2). Además, en este último grupo de derechos de agua solo se declara desierto (en el año 2006) uno de los tres remates convocados para este tipo de derechos.

Por otro lado, las convocatorias para rematar derechos de exploración de aguas subterráneas son las más frecuentes, pero también presentan el mayor porcentaje de remates declarados desiertos ( $74 \%$; ver Tabla 2). En este caso, y de forma consistente con las premisas (i) y (ii) previas sobre la hipótesis del agente especulador, el costo de presentar una solicitud rival es bajo, ${ }^{26}$ mientras que el valor de mercado del derecho solicitado puede ser elevado, dada la demanda minera por este tipo de derechos. Además, en este tipo de remates solo pueden participar los solicitantes directos de los derechos, con lo cual la premisa (iii), previa,

\footnotetext{
${ }^{25}$ La solicitud de este tipo de DAA requiere haber ya comprobado la existencia de los caudales solicitados, lo que incluye haber realizado la perforación de pozos hasta encontrar agua y demostrar que existe agua suficiente para el caudal solicitado.

${ }^{26}$ La solicitud de un derecho de exploración solo implica hacerse cargo de algunos requisitos mínimos y, eventualmente, presentar un estudio básico, pudiendo incluso copiarse parte de la información en la primera solicitud. Presentar este tipo de solicitud no necesariamente requiere realizar trabajos previos de prospección.
} 
tiende a ganar pertinencia. La Tabla A2.4 (en el Anexo 2) reporta los 13 remates que sí se realizaron para asignar nuevos derechos de este tipo. En cada uno de estos remates a lo más concurren dos participantes (en nueve de estos remates solo se presenta un agente), mientras que en la gran mayoría de ellos (12 remates) solo un participante realiza oferta (igual a $P^{M i n}$ ).

\section{Otras hipótesis}

Consideremos la opción (A), i.e. que durante el período analizado hayan ocurrido 'pocas' instancias de solicitudes rivales por un mismo DAA. ¿Qué razones podrían explicar esto?

a) Una primera posibilidad es que durante el período analizado una parte significativa del agua solicitada simplemente no haya sido escasa. Cuando el agua es muy escasa en una cuenca, la DGA emite una Declaración de Agotamiento, lo que implica que no se pueden constituir nuevos DAA en fuentes naturales de agua superficial en esa cuenca. Según la información disponible a la fecha de término de este trabajo, al año 2015 solo se habían dictado 11 declaraciones de agotamiento (cuatro en la zona norte y cinco en la zona centro). En cuencas sin declaración de agotamiento, la premisa base es que existe agua suficiente para satisfacer múltiples solicitudes de DAA. Sin embargo, aun así podría surgir escasez hídrica, y con ello solicitudes rivales, en un punto geográfico específico, gatillando el mecanismo de remate.

Las figuras $2 \mathrm{a}$ y $2 \mathrm{~b}$ muestran que durante cada uno de los años del período analizado ocurrieron regularmente asignaciones de caudales, tanto de DAA/C (de aguas superficiales y subterráneas) ${ }^{27}$ como de $\mathrm{DAA} / \mathrm{NC}$, mediante el régimen general (asignación gratuita). Sin embargo, los caudales asignados vía remate son muy bajos, en ambos tipos de DAA, en relación a los caudales totales asignados mediante el régimen general (Tabla 4, col. 3). Esta baja incidencia de asignaciones vía remate no permite descartar la hipótesis según la cual un contexto general de relativa baja escasez de DAA haya contribuido a que ocurrieran pocos remates durante el período analizado.

${ }^{27}$ En el caso de DAA/C, asignaciones de caudales mediante el régimen general de acceso ocurren en todas las regiones del país. 
Tabla 4. NUEVOS CAUDALES ASIGNADOS (M³/S), RÉGIMEN GENERAL Y REMATES. TOTAL 2005-2018

\begin{tabular}{lccr}
\hline & $\begin{array}{c}(1) \\
\text { Régimen general }\end{array}$ & $\begin{array}{c}(2) \\
\text { Remates }\end{array}$ & $\begin{array}{c}(3)=(2) /(1) \\
\%\end{array}$ \\
\hline DAA/C superficiales & 1.089 & 0,01 & 0,0009 \\
DAA/C subterráneos & 358 & 5,74 & 1,6 \\
DAA/NC (superficiales) & 39.961 & 231,1 & 0,6 \\
Total & 41.408 & 236,9 & \\
\hline
\end{tabular}

Fuente: elaboración propia en base a datos obtenidos en www.dģa.cl.

b) Otra posibilidad es que no haya sido suficiente el aumento introducido por la reforma del CA en el 2005, en el plazo para presentar solicitudes rivales (Tabla 1). Pero esta explicación no es convincente, dada la razonable extensión del nuevo plazo (seis meses desde presentada la primera solicitud) y el protagonismo que tuvo este aspecto, como parte de los debates que motivaron la reforma del año 2005.

c) Otra opción es que los costos burocráticos de llamar a remate sean demasiado onerosos para el Estado y por ello este evite este proceso. Esta explicación tampoco es razonable: los costos específicamente relacionados con la convocatoria a remate únicamente involucran gastos menores de divulgación.

d) Una cuarta posibilidad es que potenciales interesados en obtener un nuevo DAA no hayan tenido suficiente disposición a pagar para justificar el presentar una solicitud rival por un DAA ya solicitado. ¿Por qué podría ocurrir esto? ${ }^{28}$ El problema de sobreotorgamiento de DAA en determinadas cuencas puede contribuir a explicar esta posibilidad. Mientras mayor es el sobreotorgamiento en una cuenca, menor es la probabilidad de que nuevas solicitudes de DAA logren ser satisfechas. Esto crea un desincentivo a presentar nuevas solicitudes y disminuye por lo tanto la frecuencia esperada de remates de DAA.

Es un hecho que en algunas cuencas se ha producido un sobreotorgamiento de DAA (MOP 2012, 21-25; El Mercurio 2018d). Distintas

\footnotetext{
${ }^{28}$ Descartamos que un efecto de baja demanda por nuevos DAA, fruto de un ciclo económico a la baja, contribuya a explicar en forma significativa este fenómeno. Durante la década analizada, el único año con crecimiento negativo fue el 2009. Mientras que la baja ocurrencia de remates para asignar nuevos DAA es un fenómeno común a toda la década analizada.
} 
Figura 2a. CAUDALES ANUALES DE DAA/C ASIGNADOS MEDIANTE EL RÉGIMEN GENERAL (2005-2018)

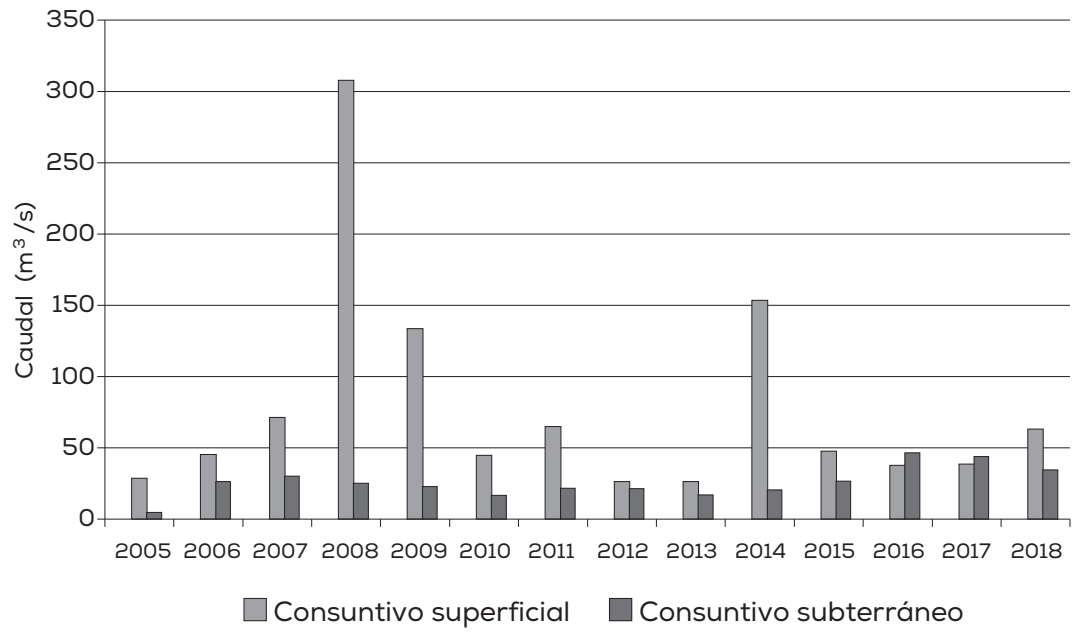

Figura $2 b$. CAUDALES ANUALES (M³/S) DE DAA/NC CONCEDIDOS MEDIANTE RÉGIMEN GENERAL (RG)

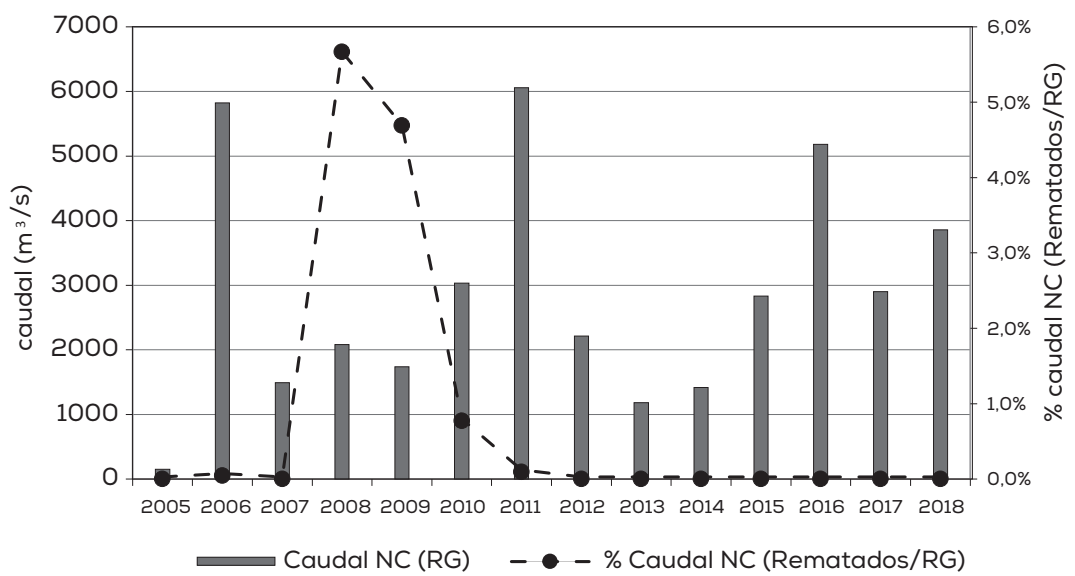

Fuente (figuras 2a y 2b): elaboración propia en base a datos obtenidos en www.dga.cl. 
razones explican este problema. En ocasiones se ha debido a falta de información. En otros casos, existiendo información, las decisiones de otorgamiento de nuevos DAA han sido técnicamente inconsistentes (e.g. en el acuífero de Copiapó; Rinaudo y Donoso 2018). De hecho, complejidades legales en el proceso para otorgar nuevos DAA, junto a la presión de grupos de interés, pueden derivar en sobreotorgamiento de DAA. Evidencia de esto es que en fuentes de aguas superficiales ya con declaración de agotamiento y en acuíferos con decreto de prohibición, el Estado continuó otorgando DAA en respuesta a un número creciente de peticiones para regularizar DAA, unido a la presión de otras agencias estatales y de grupos privados de interés (DGA 2016, DGA 2017).

Supongamos el caso de una cuenca con sobreotorgamiento de DAA. Analicemos lo que esto implicaría para distintos tipos de derechos de agua:

(d1) Aguas subterráneas: En este tipo de aguas, el problema de sobreotorgamiento de derechos es más importante (en las zonas norte y centro de Chile; McPhee et al. 2012, DGA 2016). En este tipo de caudales, la variabilidad interanual en la disponibilidad del agua es baja (relativo al caso de aguas superficiales), con lo cual contextos de sobreotorgamiento tienden a perpetuarse, mientras que los costos de acceder al agua (e.g., profundidad requerida del pozo y costos de bombeo a la superficie) aumentan en proporción a la magnitud del sobreotorgamiento. Esto reduce el interés por presentar solicitudes por nuevos derechos.

En aguas subterráneas surge un desincentivo adicional asociado al riesgo de no obtener el caudal solicitado. Solicitudes para constituir este tipo de derechos exigen realizar inversiones no despreciables, y que son costos hundidos, para alumbrar la fuente de agua por solicitar. Esto incrementa la pérdida económica en caso de no obtenerse todo el derecho solicitado, lo que debiera reducir la frecuencia de llamados a remate para asignar este tipo de aguas. Avala esta hipótesis la muy escasa ocurrencia de remates para asignar DAA para aguas consuntivas subterráneas (Tabla A2.1, Anexo 2).

(d2) Aguas superficiales: Si en una cuenca se sabe que existe sobreotorgamiento de aguas superficiales, ello reducirá el incentivo a presentar solicitudes por nuevos DAA, dada la probabilidad de no obtener todo el caudal requerido. Esta hipótesis aplica tanto a DAA/C como a 
$\mathrm{DAA} / \mathrm{NC}$, aunque en este último caso el riesgo de no obtener todo el caudal solicitado aplica con mayor fuerza a solicitudes agua abajo ${ }^{29}$ (las que son relevantes, por ejemplo, para centrales hidroeléctricas de pasada).

\section{PATRONES DE RESULTADOS EN LOS REMATES OCURRIDOS}

Esta sección ofrece algunas reflexiones sobre patrones que muestran sistematicidad en los resultados de adjudicación en los remates realizados. El objetivo de esta sección es doble. Por un lado, la descripción de estos patrones reporta información que complementa la relevancia de hipótesis analizadas en la sección precedente. Por otro, estos patrones motivan preguntas adicionales para análisis a futuro.

La Tabla 5 provee un resumen general de todos los remates que se realizaron (no declarados desiertos) durante el período 2005-2018, mientras que los Anexos 1-2 reportan información más detallada a nivel de cada remate ocurrido en este período. La Tabla 5 reporta información según el tipo de derecho de agua en oferta (categorías a hasta d). En el caso de DAA/C, se distingue entre aguas superficiales y subterráneas. Además, los remates se clasifican según rangos de valor del indicador $R$ (definido como valor promedio, por remate ocurrido), que se calcula del siguiente modo: usando los precios unitarios de adjudicación ( $P^{a}$, expresado en $\$ / \mathrm{m}^{3} / \mathrm{s}$ ), obtenidos al vender cada cuota adjudicada, primero se calcula el indicador $R_{c}=$ $\left(P_{c}^{a} / P_{c}^{M i n}\right) \geq 1$ en donde $P_{c}^{a}$ es el precio unitario de adjudicación y $P_{c}{ }^{M i n}$ el correspondiente precio mínimo de postura (ambos por cuota $c$ rematada). A continuación, se calcula el indicador $R$, ahora como valor promedio por remate, ponderando los distintos $R_{c}$ (calculados para cada cuota adjudicada en un determinado remate), según la proporción que representa el caudal de cada cuota $c$ en el caudal total asignado en el respectivo remate.

Los rangos de valor considerados para el indicador $R$ en la Tabla 5 tienen la siguiente lógica: la columna (1) se refiere a los remates en

\footnotetext{
${ }^{29}$ En el caso de aguas no consuntivas en zonas de cabecera de cuenca hidrográfica (solicitud 'aguas arriba'), los solicitantes debieran saber con mayor certeza si la zona de interés está ya otorgada o no y, por lo tanto, el argumento de incerteza asociada a un contexto de sobreotorgamiento tiende a perder relevancia.
} 
donde todas las cuotas rematadas fueron adjudicadas al precio mínimo de oferta $\left(P^{M i n}\right)$. Los otros dos rangos de valor considerados para $R$ (columnas 2 y 3 ) guardan relación con el criterio que la DGA sistemáticamente ha usado para calcular el $P^{\text {Min }}$ de cada cuota en oferta: esto es, $P^{\mathrm{Min}}=(0,1 \cdot V C)$, donde $V C$ es el valor comercial de referencia que la propia DGA asigna a cada cuota. Así, los casos con $R>10$ se refieren a remates en donde los precios unitarios de adjudicación $\left(P^{a}\right.$, en $\$ / \mathrm{m}^{3} / \mathrm{s}$ ), de las distintas cuotas ofertadas en ese remate, en promedio superaron a sus respectivos $V C$. La columna (2) reporta el resto de casos intermedios.

Esta clasificación permite visualizar en forma resumida la distribución de los remates ocurridos y de características asociadas a ellos, según rangos de magnitud de diferencia entre los precios (unitarios) de adjudicación, promedio por remate, y los respectivos $P^{\text {Min }}$. Dados los criterios de valoración que la DGA usa para definir $P^{\text {Min }}$, el indicador $R$ informa cuánto divergen en promedio, por sobre sus respectivas valoraciones $P^{M i n}$, los precios de adjudicación que resultan de las interacciones de mercado que ocurren en cada remate.

A continuación, algunos patrones recurrentes que se constatan en la muestra de remates descritos en la Tabla 5:

1) Resalta la recurrencia de remates con muy pocos participantes. La Tabla 5 reporta el número de remates con $P \leq 2$ ( $P$ : número de participantes por remate). Del total de 58 remates ocurridos, 38 de ellos (66\%) comparten esta condición. En el grupo de remates con indicador $R=1$ (34 en total), 28 (82\%) de ellos cumplen esta condición. De hecho, en $23(68 \%)$ de los remates con indicador $R=1, P=1$ (detalles en Anexos 1-2). En el total de nuestra muestra de remates realizados, el número promedio de participantes por remate fue de 2,3.

2) La tasa de incidencia de remates con indicador $R=1$ es alta: esto ocurre en 34 (59\%) del total de (58) remates realizados. Ya vimos que en la mayoría ( $82 \%$ ) de estos remates ocurre que $P \leq 2$. Además, el resultado $R=1$ ocurre en un porcentaje mayoritario de los remates de derechos distintos de aguas (solo) no consuntivas. De hecho, $R=1$ ocurre: en el $100 \%$ de los remates 'mixtos' y de DAA/C de aguas subterráneas (Tabla A2.3, Anexo 2); en 11 de los 12 remates realizados para asignar superficies de exploración para aguas subterráneas (Tabla A2.4, Anexo 
Tabla 5. RESUMEN DEL TOTAL DE REMATES OCURRIDOS (DESDE INICIOS DE JUNIO DE 2005 HASTA FINES DE DICIEMBRE DE 2018)

\begin{tabular}{|c|c|c|c|c|c|}
\hline & & $\begin{array}{l}(1) \\
R=1\end{array}$ & $\begin{array}{c}(2) \\
1<R \leq 10\end{array}$ & $\begin{array}{c}(3) \\
R>10\end{array}$ & $\begin{array}{l}\text { (4) } \\
\text { Total }\end{array}$ \\
\hline \multicolumn{6}{|c|}{ a) DAA/C } \\
\hline \multirow[t]{2}{*}{$\begin{array}{l}\text { Número de } \\
\text { remates }\end{array}$} & $\begin{array}{l}\text { Aguas subterráneas } \\
\text { \{remates con } \mathrm{P} \leq 2 \text { \} }\end{array}$ & $\begin{array}{c}2 \\
\{1\}\end{array}$ & 0 & 0 & $\begin{array}{c}2 \\
\{1\}\end{array}$ \\
\hline & $\begin{array}{l}\text { Aguas superficiales } \\
\text { \{remates con } P \leq 2\}\end{array}$ & $\begin{array}{c}4 \\
\{4\}\end{array}$ & 0 & $\begin{array}{c}4 \\
\{4\}\end{array}$ & $\begin{array}{c}8 \\
\{8\}\end{array}$ \\
\hline \multicolumn{6}{|c|}{ Total caudal rematado $\left(\mathrm{m}^{3} / \mathrm{s}\right)^{* *}$} \\
\hline & Aguas subterráneas & 5,74 & & & 5,74 \\
\hline & Aguas superficiales & 0,0056 & & 0,0046 & 0,01 \\
\hline \multicolumn{6}{|c|}{ Valor total recaudación (\$ MM) } \\
\hline & Aguas subterráneas & 188,8 & & & 188,8 \\
\hline & Aguas superficiales & 0,18 & & 34 & 34,2 \\
\hline
\end{tabular}

Precio unitario promedio ${ }^{+}$de adjudicación (\$ MM $/ \mathrm{m}^{3} / \mathrm{s}$ )

Aguas subterráneas $\quad 32,9(0.13)$ *

Aquas superficiales $\quad 0,02(0,0002)$ * $6,3(0,7) *$

b) Remates con DAA 'mixtos'

Número de remates

8

0

\{remates con $\mathrm{P} \leq 2$ \}

\{3\}

$\{3\}$

Total caudal rematado $\left(\mathrm{m}^{3} / \mathrm{s}\right)$

Valor total recaudación (\$ MM)

404,7

404,7

Precio unitario promedio ${ }^{+}$de adjudicación $\left(\$ \mathrm{MM} / \mathrm{m}^{3} / \mathrm{s}\right)$

$4,4(1,06)$ *

\section{c) Superficies de exploración (aguas subterráneas)}

Número de remates 12

$\{1\}$

\{remates con $\mathrm{P} \leq 2$ \}

65.788

4.645

Superficie total rematada $(\mathrm{Ha})$

308,4

101,2

70.433

Valor total recaudación (\$ MM)

0,005

409,6

Precio unitario promedio ${ }^{++}$de adjudicación (\$ MM/Ha)

\begin{tabular}{|c|c|c|c|c|}
\hline \multicolumn{5}{|c|}{ d) $\mathrm{DAA} / \mathrm{NC}$} \\
\hline $\begin{array}{l}\text { Número de remates } \\
\text { \{remates con } P \leq 2 \text { \} }\end{array}$ & $\begin{array}{c}8 \\
\{8\}\end{array}$ & $\begin{array}{c}11 \\
\{5\}\end{array}$ & $\begin{array}{c}8 \\
\{0\}\end{array}$ & $\begin{array}{c}27 \\
\{13\}\end{array}$ \\
\hline Total caudal rematado $\left(\mathrm{m}^{3} / \mathrm{s}\right)$ & 9,34 & 70,04 & 151,72 & 231,1 \\
\hline $\begin{array}{l}\text { Caudal promedio adjudicado, por } \\
\text { remate }\left(\mathrm{m}^{3} / \mathrm{s}\right)\end{array}$ & 1,2 & 6,4 & 18,9 & \\
\hline Valor total recaudación (\$ MM) & 163,1 & $1.269,5$ & $47.081,6$ & $48.514,2$ \\
\hline $\begin{array}{l}\text { Precio unitario promedio }{ }^{+} \text {de } \\
\text { adjudicación }\left(\$ \mathrm{MM} / \mathrm{m}^{3} / \mathrm{s}\right)\end{array}$ & $\begin{array}{c}17,5 \\
(3,3)^{*}\end{array}$ & $\begin{array}{c}18,1 \\
(1,8) *\end{array}$ & $\begin{array}{c}310,3 \\
(58,6) *\end{array}$ & \\
\hline
\end{tabular}

Notas: $\mathrm{P}=$ número de participantes por remate.

*: Valores entre paréntesis (.) = desviación estándar del precio unitario promedio de adjudicación; ${ }^{* \star}: 1 \mathrm{~m}^{3}$ de agua $\cong 10^{3}$ litros; $^{+}$: promedio ponderado por volumen de caudal rematado; ${ }^{++}$: promedio ponderado por superficie rematada.

Fuente: elaboración propia en base a datos obtenidos en www.dga.cl. 
2); y en la mitad de los remates de DDA/C de aguas superficiales (Tabla A2.1, Anexo 2)..$^{30}$

3) En los remates de DDA/NC (grupo d en Tabla 5), la incidencia del resultado $R=1$ es claramente menor (que en los remates del resto de derechos de agua): la condición $R=1$ se cumple en 8 de los 27 remates realizados para DAA/NC. De hecho, 2/3 del volumen total de caudales de DAA/NC rematados se adjudicaron en remates con indicador $R>10$. Es posible sugerir al menos tres factores condicionantes que pueden ayudar a entender este tercer patrón:

i) Los $\mathrm{DAA} / \mathrm{NC}$ normalmente tienen un mayor valor (unitario) de mercado que los DAA/C (aguas superficiales).

ii) En los remates de DAA/NC se adjudicaron volúmenes (caudales) de agua muy superiores a los caudales rematados de DAA/C. Relacionado con esto, nótese que, dentro de los propios remates de DAA/NC, mientras mayores son los caudales de agua adjudicados (promedio por remate), mayores tienden a ser los valores de los correspondientes indicadores $R$.

iii) Desde la reforma del CA en el año 2005, en remates de aguas superficiales puede participar cualquier interesado, no solo los solicitantes de los derechos (ver Tabla 1). Esto pudo haberse reflejado en un relativo mayor número de participantes en los remates de DAA/NC (para detalles, ver Anexo 1; remates con indicador $R>1$ ).

Estos patrones motivan algunas preguntas. Primero, ¿por qué es recurrente el patrón de muy pocos participantes en los remates ocurridos? Es muy probable que parte de las hipótesis que analizamos en la sección 6 sean aquí también relevantes. En particular, lo discutido respecto de: (a) el accionar de agentes especuladores; (b) condiciones que facilitarían acuerdos ex ante entre los potenciales participantes en un remate; y (c) las otras conjeturas relacionadas con la posibilidad de que prevalezcan, en determinados contextos (e.g., sobreotorgamiento de

\footnotetext{
${ }^{30}$ En el caso de DAA/C para aguas subterráneas se requiere 'hundir' costos no despreciables al realizar solicitudes, lo que podría entregar ventajas de 'primer movimiento' al primer solicitante. Además, en aguas subterráneas el problema de sobreotorgamiento es más importante, lo que reduce el número de interesados en participar. En el caso de solicitudes sobre derechos de exploración de aguas subterráneas, los incentivos para el accionar de especuladores pueden ser más altos que en otros tipos de DAA, lo que podría derivar en acuerdos entre potenciales participantes, previos a la realización del remate.
} 
derechos), bajas disposiciones de pago para adquirir nuevos derechos de agua a través del sistema de remates.

En segundo lugar, ¿de qué forma pueden haber influido decisiones de diseño licitatorio en la recurrencia de los patrones aquí descritos? Estas decisiones involucran definiciones sobre el mecanismo usado para licitar los derechos en venta (e.g., en el caso analizado, remates orales y venta secuencial de múltiples cuotas por remate; conocimiento ex ante de precio mínimo de oferta); y también definiciones sobre los objetos en venta (e.g., numerosas cuotas ofertadas por remate; $\mathrm{y}$, en varios remates, múltiples cuotas con muy pequeños caudales en oferta). Por ejemplo, el conocimiento público ex ante del precio mínimo de oferta $\left(P^{M i n}\right)$ — con hasta dos meses de anticipación por parte de los posibles interesados en participar en el remate- puede facilitar acuerdos colusorios entre los interesados. Asimismo, la venta secuencial y a viva voz de numerosas cuotas en oferta, en particular cuando el número de participantes es reducido, es otro factor que favorece el desarrollo de acuerdos de coordinación entre los participantes en un remate. ${ }^{31}$

Dejamos planteadas estas interrogantes para análisis a futuro. Desarrollar aquí un análisis detallado sobre estas preguntas escapa al ámbito y objetivos de este primer trabajo exploratorio sobre la experiencia chilena en el uso de remates para resolver la asignación inicial de distintos tipos de derechos de agua.

El análisis de estas preguntas requiere tener en cuenta múltiples condicionantes. Además, lo ideal es considerar información detallada a nivel de cada cuota rematada. Esto último, dada la heterogeneidad de los objetos (cuotas) en venta, las características idiosincráticas de los intereses de participación en estos remates (e.g., accionar reiterado de agentes especuladores) y el conjunto (complejo) de estrategias posibles de oferta y los múltiples posibles equilibrios resultantes (dada

\footnotetext{
${ }^{31}$ Los Anexos 1-2 reportan información, por remate realizado, sobre el número de participantes y el número de cuotas en oferta. Un resultado consensuado en teoría de subastas es que mientras mayor es el número de objetos en venta, respecto del número de participantes en la subasta, más probable será, ceteris paribus, que los participantes logren alcanzar algún mecanismo de coordinación que les permita reducir la intensidad de rivalidad entre ellos; sobre todo cuando los participantes son pocos y las ofertas son orales (Klemperer 2004, 2008; Salmon 2004; Hoppe, Jehiel y Moldavanu 2006; Hendricks y Porter 2007; Peña-Torres y Fernández 2010).
} 
la venta secuencial de múltiples cuotas y remates que se suceden en el tiempo, que podrían involucrar cuotas en oferta con valoraciones interdependientes). ${ }^{32}$

\section{CONCLUSIONES}

En el período analizado ocurrieron muy pocos remates de nuevos derechos de agua. Los caudales así asignados representaron menos del $1 \%$ del total nacional de nuevos derechos de agua asignados durante 2005-2018. Más de la mitad (57\%) de los remates convocados fueron declarados desiertos. $\mathrm{Y}$ en los remates que ocurrieron con frecuencia asistieron pocos participantes. Además, en los remates con pocos participantes predominaron adjudicaciones a los precios mínimos de oferta.

¿Por qué no se han cumplido las expectativas de los promotores de la reforma del CA del año 2005, que buscaba dinamizar el uso de remates como mecanismo asignador de nuevos derechos de agua?

Como vimos, una primera posibilidad es que durante el período analizado una parte significativa del agua solicitada simplemente no haya sido escasa. Dados los significativos caudales de agua que fueron asignados a través del régimen general (asignación gratuita) durante el período analizado, no podemos descartar la validez de esta hipótesis como una de las posibles causas.

Un segundo factor causal es que, desde hace varios años, existe sobreotorgamiento de derechos de agua en algunas cuencas. Esto crea desincentivos a presentar nuevas solicitudes y disminuye la frecuencia esperada de que ocurran remates en esas cuencas.

Una tercera posibilidad es que parte de los solicitantes de nuevos derechos hayan desistido de su solicitud o hayan desistido de competir por la adjudicación del derecho solicitado. Así se podrían haber abortado llamados a remate (desistimiento de solicitudes). O bien, si la convocatoria al remate ya hubiese ocurrido, que el remate fuese declarado desierto (no asistencia de los solicitantes), o que los derechos rematados se hayan adjudicado al precio mínimo de puja (con

32 Ejemplos de análisis empírico sobre subastas con este tipo de desafíos conceptuales: Hendricks y Porter 1988, 2007; Porter y Zona 1993; Athey, Levin y Seira 2011; Athey y Levin 2001; Baldwin, Marshall y Richard 1997. 
solo un participante efectuando oferta). Como vimos, este conjunto de resultados tiene dos orígenes posibles. Podrían ser consecuencia de (i) acuerdos de coordinación (ex ante a la ocurrencia del remate) entre los interesados en participar en un remate, para reducir el precio de adjudicación del derecho de agua, o resultado de (ii) solicitudes presentadas por agentes especuladores para obtener una renta a través de crear una solicitud rival artificial respecto de un DAA solicitado por otro agente.

Un cuarto factor causal se relaciona con decisiones y operatividad administrativa dentro de la DGA. En el proceso de resolución de solicitudes por nuevos DAA, se pueden acumular tiempos de respuesta de la DGA que en la práctica exceden los plazos máximos de respuesta (no legalmente exigibles), establecidos en el propio CA. En una proporción significativa de casos, la resolución de este proceso se ha prolongado por varios años.

En tanto siga siendo válido el objetivo de dinamizar el uso de remates o licitaciones como mecanismo asignador de nuevos derechos de agua, las conjeturas analizadas en este trabajo plantean distintos desafíos por resolver. Como parte de estos desafíos, falta analizar el rol jugado por las definiciones de diseño licitatorio que se han implementado para asignar nuevos derechos de agua mediante remate.

\section{REFERENCIAS}

Athey, S. y Levin, J. 2001. Information and Competition in US Forest Timber Auctions. Journal of Political Economy 109 (2), 375-417.

Athey, S., Levin, J. y Seira, E. 2011. Comparing Open and Sealed Bid Auctions: Evidence from Timber Auctions. Quarterly Journal of Economics 126, 207-57.

Baldwin, L., Marshall, R. y Richard, J-F. 1997. Bidder Collusion at Forest Service Timber Sales. Journal of Political Economy 105 (4), 657-99.

Bauer, C.J. 1997. Bringing Water Markets down to Earth: The Political Economy of Water Rights in Chile, 1976-95. World Development 25 (5), 639-56.

Comisión Especial Mixta de Presupuesto del Senado 2016. Informe aclaratorio Seguimiento de compromisos de Ley de Presupuesto del Sector Público. Santiago: Ministerio de Obras Públicas.

Congreso Nacional de Chile 2005. Historia de la Ley $N^{\circ} 20.017$ (que modificó el Código de Aguas en el año 2005). Congreso Nacional de Chile.

Dardati, E. y De Elejalde, R. 2015. ¿Cómo funciona el mercado de certificados verdes en Chile? Observatorio Económico 94. Disponible en: http:/fen. 
ua/hurtado.cl/2015/articulos/observatorio-economico/como-funciona-elmercado-de-certificados-verdes-en-chile/.

DGA 2016. Atlas del Agua, Chile. Disponible en: http://www.dga.cl/atlasdelagua/ Paginas/default.aspx.

DGA 2017. Derechos de Aguas. Disponible en: http://www.dga.cl/sitioDerechos/ Paginas/default.aspx.

El Mercurio 2017. Derechos de agua con remates pendientes por hasta una década suman US\$60 millones y Gobierno busca agilizar subastas. El Mercurio, 20 de agosto 2017, B7.

El Mercurio 2018a. Patentes de agua impagas. Editorial. El Mercurio, 19 de abril 2018, A3.

El Mercurio 2018b. Morosidad de patentes por no uso de derechos de agua llega a récord y acumula US\$317 millones. El Mercurio, 5 de agosto 2018, B16.

El Mercurio 2018c. Cobros por no uso de derechos de agua. Editorial. El Mercurio, 15 de agosto 2018, A3.

El Mercurio 2018d. Gobierno define acciones para garantizar seguridad hídrica y frenar sobreexplotación de cuencas. Economía y Negocios. El Mercurio, 16 de octubre 2018.

Gentes, I. 2006. El status jurídico y el debate entre derechos individuales y colectivos de aguas. Análisis de legislación, políticas hídricas y jurisprudencia sobre derechos de aguas y gestión ciudadana en Chile. Informe. Santiago: Comisión para la Gestión Integral de Agua en Bolivia, GIAB; Centro Internacional de Investigaciones para el Desarrollo, IDRC, Canadá, Proyecto Visión Social del Agua.

Hendricks, K. y Porter, R. 1988. An Empirical Study of an Auction with Asymmetric Information. American Economic Review 78 (5), 865-83.

Hendricks, K. y Porter, R. 2007. An Empirical Perspective on Auctions. En Handbook of Industrial Organization, editado por M. Armstrong y R. Porter. Amsterdam: Elsevier, Vol. 3, cap. 32, 2073-143.

Hoppe, H., Jehiel, P. y Moldovanu, B. 2006. License Auctions and Market Structure. Journal of Economics \& Management Strategy 15, 371-96.

Klemperer, P. 2004. Auctions: Theory and Practice. Princeton, NY: Princeton University Press.

Klemperer, P. 2008. Competition Policy in Auctions and Bidding Markets. En Handbook of Antitrust Economics, editado por P. Buccirossi. Cambridge, MA: The MIT Press, cap. 16, 583-624.

Krishna, V. 2002. Auction Theory. London: Academic Press.

Lagos, R. 1997. Discusión en Cámara de Diputados, Legislatura 334, Sesión 69, mayo 13. Historia de la Ley $\mathrm{N}^{\circ}$ 20.107. Congreso Nacional de Chile.

McPhee, J., De la Fuente, A., Herrera, P., Niño, Y., Olivares, M., Sancha, A.M., Tamburrino, A. y Vargas, X. 2012. El sector del agua en Chile: su estado y sus retos. En Diagnóstico del agua en las Américas, editado por B. Jiménez Cisneros y J. Galizia Tundisi. Foro Consultivo Científico y Tecnológico, AC. 
México. Disponible en: https://www.ianas.org/water/book/diagnostico_del_ agua_en_las_americas.pdf.

Ministerio de Obras Públicas 2012. Estrategia Nacional de Recursos Hídricos 20122025. Santiago DGA/MOP. Disponible en: https://www.mop.cl/Documents/ ENRH_2013_OK.pdf.

Peña, H., Luraschi, M. y Valenzuela, S. 2004. Agua, desarrollo y políticas públicas. Estrategias para la inserción del agua en el desarrollo sostenible. Documento de Discusión. Comité Asesor Técnico de América del Sur (SAMTAC), Asociación Mundial para el Agua (Global Water Partnership, GWP). Disponible en: https://www.cepal.org/ilpes/noticias/paginas/1/35691/ PenaLuraschiValenz.pdf.

Peña-Torres, J. y Fernández, G. 2010. Disuasión de entrada, subastas repetidas y divisibilidad del objeto en venta. Estudios de Economía 37 (1), 105-49.

Porter, R. y Zona, J.D. 1993. Detection of Bid Rigging in Procurement Auctions. Journal of Political Economy 101, 518-38.

Riestra, F. 2008. Pago de patente por no uso de derechos de aprovechamiento. Santiago: DGA/MOP.

Rinaudo, J.D. y Donoso, G. 2018. State, Market or Community Failure? Untangling the Determinants of Groundwater Depletion in Copiapó (Chile). International Journal of Water Resources Development 35 (2), 283-304.

Salant, D. 2014. A Primer on Auction Design, Management and Strategy. Cambridge, MA: The MIT Press.

Salmon, T.C. 2004. Preventing Collusion among Firms in Auctions. En Auctioning Public Assets. Analysis and Alternatives, editado por M.C.W. Janssen. Cambridge, UK: Cambridge University Press, cap. 3, 80-107.

TDLC 2007. Resolución $\mathrm{N}^{\circ}$ 22. Proceso de Consulta (Rol NC No 134-06) de empresas Endesa S.A. y Colbún S.A. sobre alianza para realización de proyecto hidroeléctrico Aysén. Santiago.

Weisner, R. 2007. Entrevista: El agua, derechos y deberes. Capital, 23 de marzo 2007. Disponible en https://www.capital.cl/el-agua-derechos-y-deberes/.

Wolfstetter, E. 1999. Topics in Microeconomics. Industrial Organization, Auctions and Incentives. Cambridge: Cambridge University Press. 


\section{ANEXOS}

Los Anexos 1 y 2 reportan información a nivel de cada remate ocurrido en el período analizado. Los remates se clasifican según las categorías consideradas en la Tabla 5. Estos anexos complementan la información que se analiza en este artículo. Además, se reportan como aporte para análisis futuro sobre el tema. En cada remate se informa el respectivo indicador $R$ (valor promedio por remate) y otros dos indicadores, $P$ y $Z$, relacionados con el número de participantes por remate.

En licitaciones con múltiples unidades (cuotas, en nuestro caso) del producto en oferta, interesa conocer, como paso inicial para analizar el tipo de interacciones de mercado que pudo haber prevalecido entre los múltiples participantes por remate: (1) si los oferentes demandan múltiples unidades (o no) y (2) qué relación prevalece entre el número de participantes, dadas sus demandas por los objetos en oferta, y el número de objetos en oferta (Salant 2014, Krishna 2002, Wolfstetter 1999). Al respecto, un primer indicador básico es $Z=(P / G)$, donde $P$ indica el número de participantes (por remate) y $G$ el número de ellos que lograron adjudicarse al menos una cuota rematada. Así, conocido $P$, el indicador $Z$ informa el número de participantes que logran adjudicaciones de cuota y el número de los que no logran ninguna asignación.

\section{Anexo 1: Remates de DAA/NC}

a) DAA/NC: remates con indicador $\mathrm{R}=1$

En la mayoría de los ocho remates (salvo uno) de este tipo, se presenta un único postor y siempre todas las cuotas rematadas se adjudican a $P^{M i n}$. En el único remate (\#2) con dos participantes, todas las 55 cuotas allí rematadas fueron adjudicadas a un mismo y único postor, pagando $P^{\text {Min }}$. La mayoría de los caudales asignados en estos remates tienen potencial de generación eléctrica (columna 10). ${ }^{33}$

\footnotetext{
${ }^{33}$ En el caso del mercado eléctrico, el límite inferior de generación de planta es 0,1 MW para microcentrales de pasada que aportan al sistema interconectado central (SIC). Fuente: sitio web CEDEC.
} 


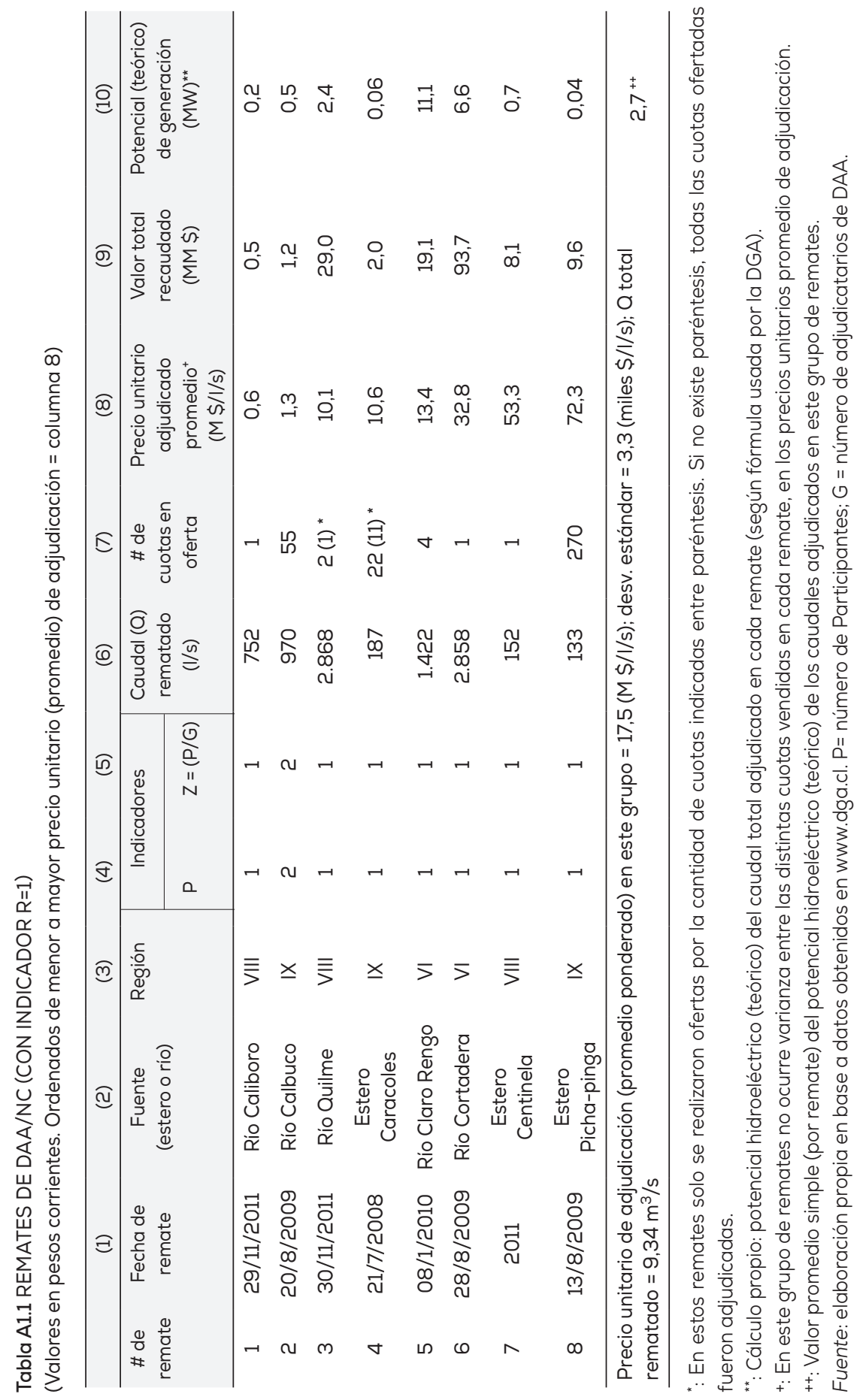




\section{b) DAA/NC: remates con indicador $1<R \leq 10$}

En la mayoría de estos remates, salvo dos de ellos (\#8 y 10), las distintas cuotas adjudicadas, en un remate dado, son adquiridas por un único postor, a pesar de que siempre existe más de un participante. En los remates con mayor número de cuotas en oferta (remates $\# 2,8,10$ ), no todas las cuotas pudieron adjudicarse. Los dos remates (\#1 y 2) que en este grupo alcanzan los mayores valores del indicador $\mathrm{R}$, corresponden a derechos de agua solicitados para uso en piscicultura (ver Tabla A1.2).

\section{c) DAA/NC: remates con indicador $R>10$}

En estos remates, el promedio del potencial hidroeléctrico de los caudales adjudicados supera el caudal promedio en las Tablas A1.1 y A1.2. Esto probablemente contribuye a explicar que en este tercer grupo de remates de DAA/NC se obtengan valores más altos para el indicador $R$. En este grupo de remates, todas las cuotas ofertadas fueron adjudicadas. En los remates en que se ofertan múltiples cuotas, las cuotas con los mayores caudales en oferta tienden a obtener los mayores valores del respectivo indicador $R_{c}$. Además, en este grupo de remates se observa como resultado mayoritario (en cuatro de los siete remates con multiples cuotas) que todas las cuotas ofertadas, en un determinado remate, son adjudicadas a un único postor (distinto en cada remate) $)^{34}$ (ver Tabla A1.3).

\section{Anexo 2: Remates de DAA distintos de aguas no consuntivas}

Un porcentaje mayoritario de los remates descritos en este anexo presentan indicadores $\mathrm{R}=1$. La excepción (parcial) es el grupo de remates de $\mathrm{DAA} / \mathrm{C}$ de aguas superficiales.

\section{d) DAA/C aguas superficiales}

En los remates \#1 al \#4, el precio de adjudicación promedio por remate superó con creces el valor de referencia $(V C)$ estimado por la DGA $(R>10)$. En estos cuatro remates siempre participaron los mismos dos agentes, aunque solo uno de ellos (siempre el mismo agente) se adjudicó todas las cuotas rematadas. Estos cuatro remates corresponden a caudales en un mismo sector geográfico. En los otros cuatro remates (\#5 al \#8), todas las cuotas rematadas fueron adjudicadas a $P^{M i n}$ (ver Tabla A2.1).

\footnotetext{
${ }^{34}$ En los otros tres remates (\#3, 5 y 7), las cuotas adjudicadas se reparten entre dos ganadores (distintos en cada remate).
} 


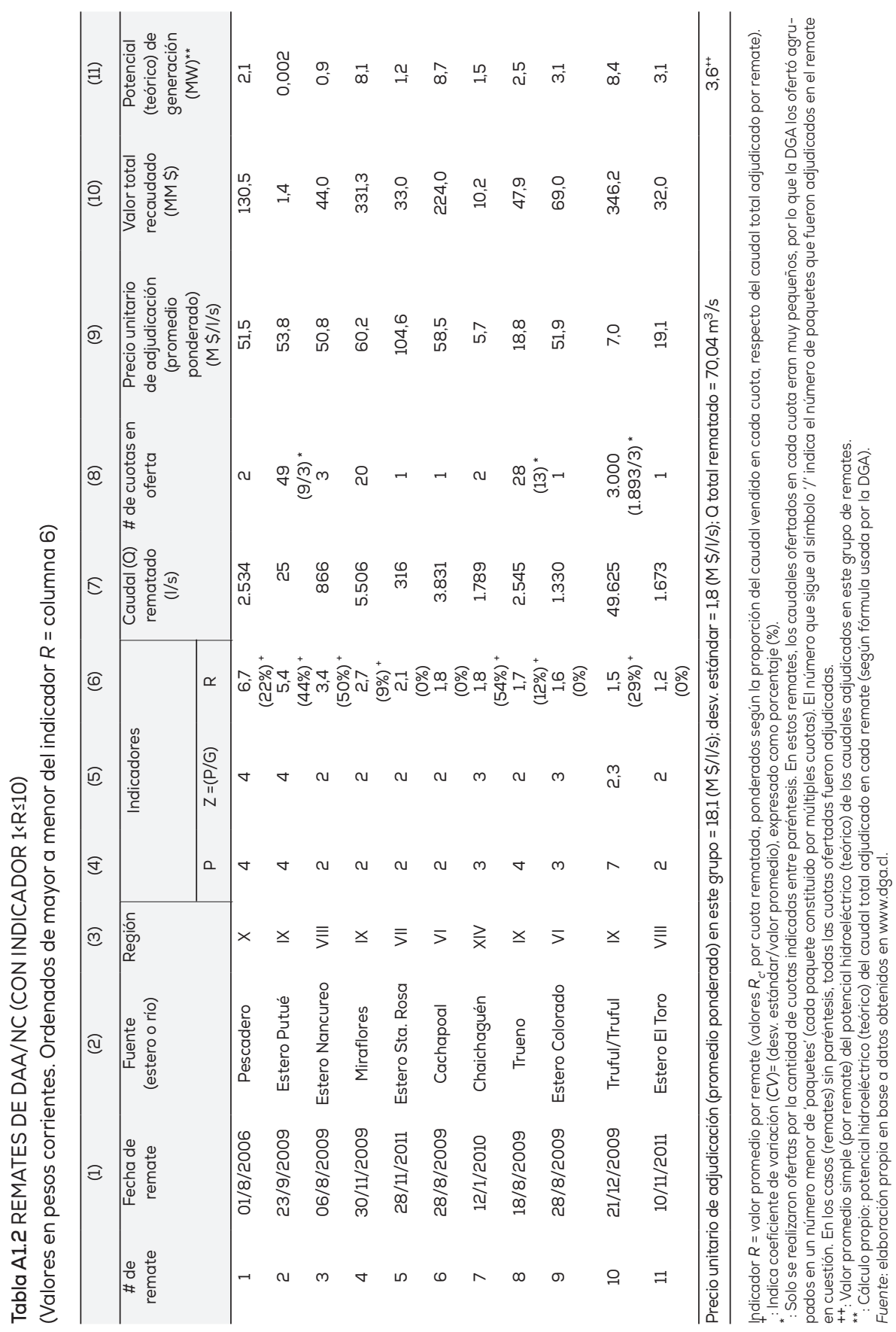




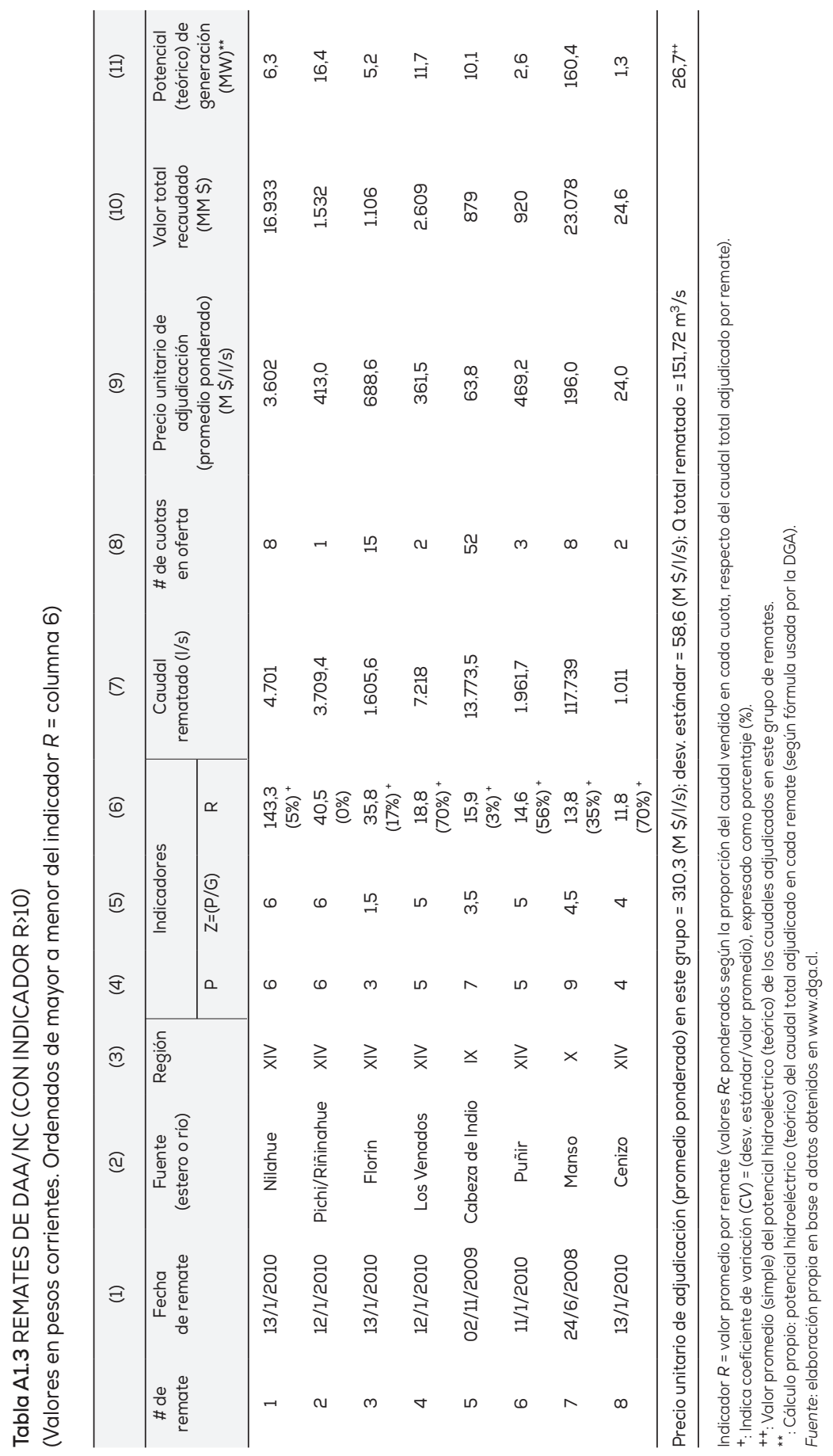




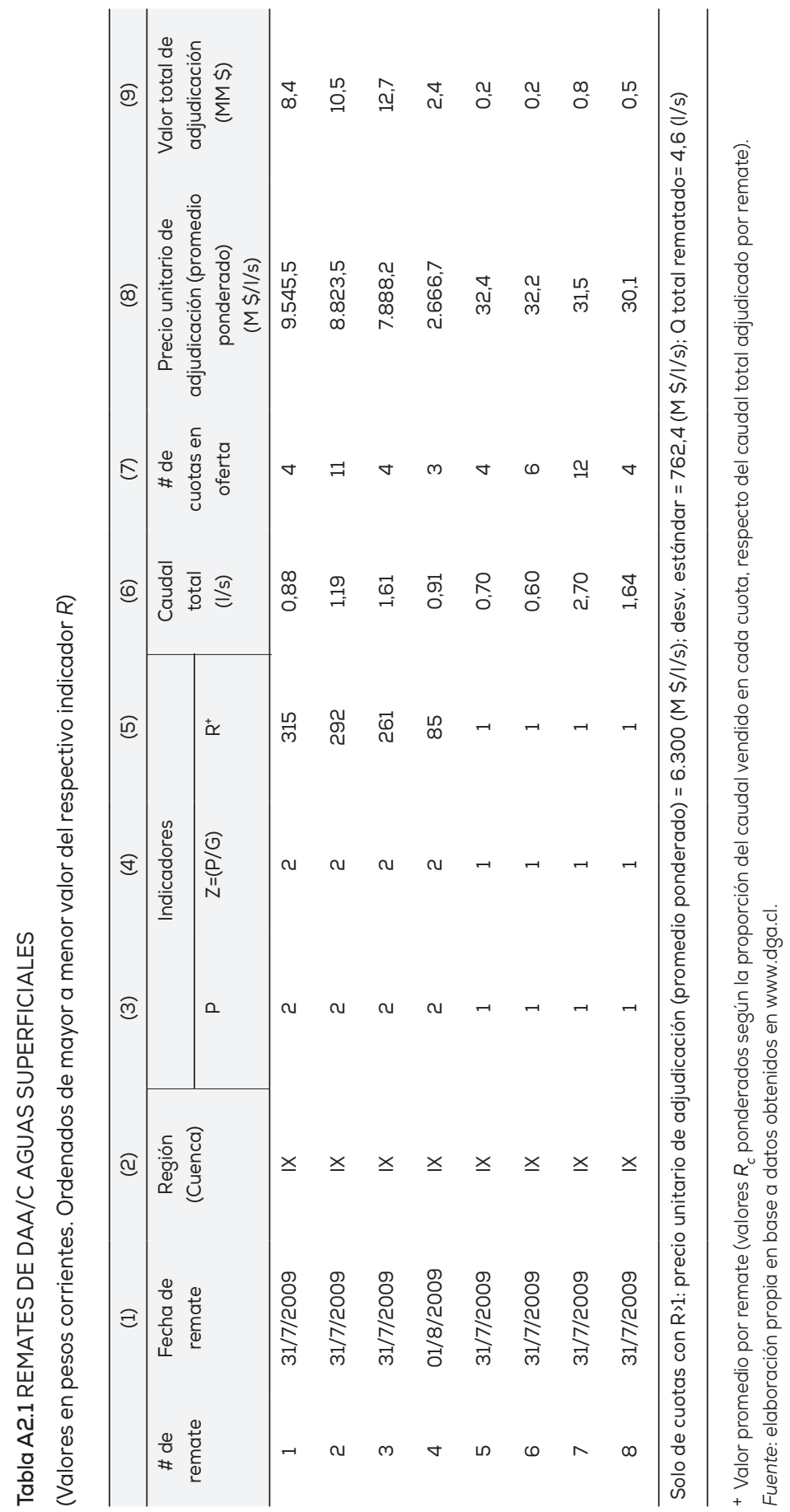




\section{e) DAA/C aguas subterráneas: todos con indicador $R=1$}

En esta categoría se registran solo dos remates realizados. En ambos, todas las cuotas fueron adjudicadas a $P^{M i n}$. El primer remate (año 2007) se produjo a raíz de varios caudales solicitados para uso agrícola; los caudales solicitados eran parte de un sector hidrogeológico común en la VI Región. La DGA decidió vender en un mismo remate los distintos DAA solicitados. En total se remataron 547 cuotas y cada uno de los ocho participantes, coincidentes con los ocho solicitantes originales de los DAA, obtuvo cuotas. Las cuotas así asignadas correspondían a caudales subterráneos en terrenos privados de cada solicitante. Los caudales se adjudicaron al respectivo solicitante original y cada uno pagó $P^{M i n}$.

El segundo remate se produjo en la Región de Antofagasta: se remataron 410 cuotas, dentro de un sector hidrogeológico común en el desierto de Atacama. Solo se presentó un participante (minera Xstrata), obteniendo todas las cuotas a $P^{\text {Min }}$ (Tabla A2.2).

\section{f) DAA mixtos}

En esta categoría se efectuaron ocho remates. Prácticamente todas las cuotas adjudicadas se vendieron a $P^{M i n 35}$ (Tabla A.2.3).

\section{§) Remates de derechos para exploración de aguas subterráneas}

El objeto rematado es un permiso temporal (dos años) para realizar prospecciones hidrogeológicas en terrenos fiscales para determinar la existencia de un acuífero explotable. La zona norte de Chile es propicia para solicitar este tipo de derecho: extensas superficies de propiedad fiscal, escasez de agua para otorgar nuevos derechos y alta demanda del recurso (sobre todo para minería).

Los remates realizados en este grupo fueron 13; en 12 de ellos se obtiene un indicador $R=1$. En 9 de esos 12 remates solo asistió un solicitante, pagando $P^{\text {Min }}$ para adquirir los derechos en oferta. En los otros tres remates asistieron dos participantes por remate, pero en cada caso solo uno de ellos realizó oferta (la puja mínima $P^{M i n}$ ). En el único remate (\#1) en donde el precio de adjudicación superó a $P^{M i n}$, los solicitantes fueron la minera SQM Salar y Asesorías e Inversiones Huturi S.A. (comercializadora de derechos de agua). La adjudicataria fue Huturi (Tabla A.2.4).

\footnotetext{
${ }^{35} \mathrm{El}$ remate \#1 obtiene un indicador $R$ levemente superior a 1, porque 1 de las 19 cuotas allí adjudicadas se vendió a un precio superior a su $P^{\text {Min }}$.
} 
J. PEÑA-TORRES, E. VARGAS y G. DONOSO / Remate de derechos de agua en Chile 101

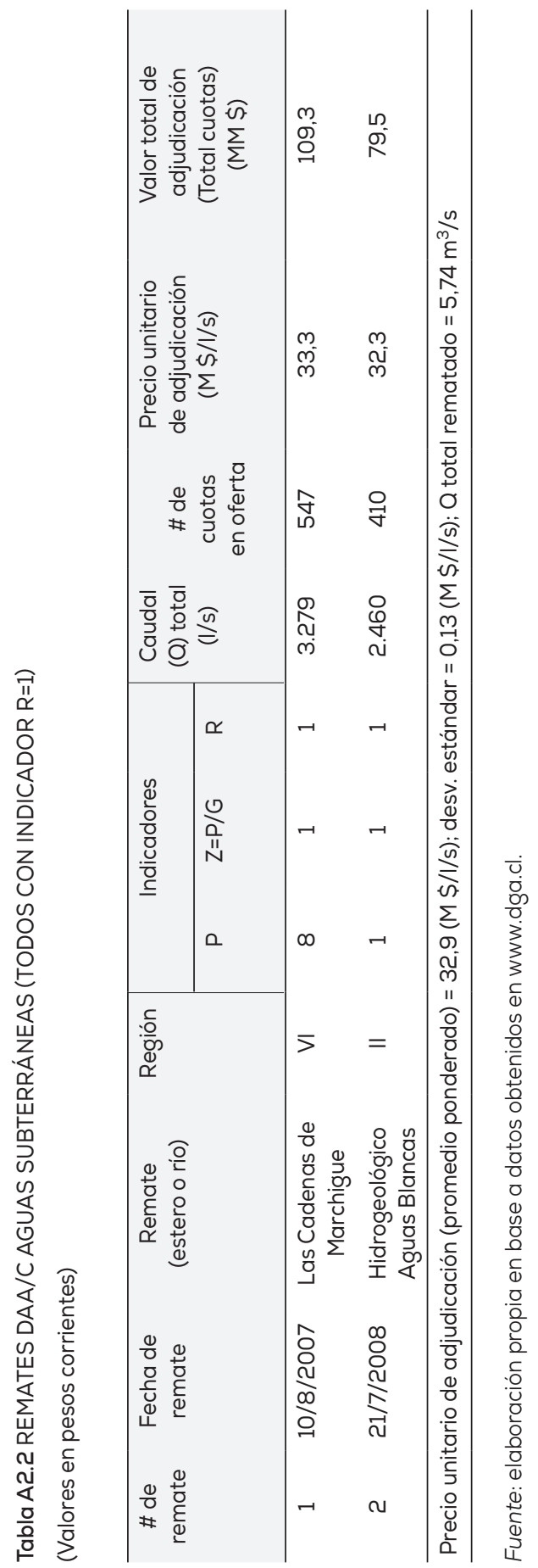




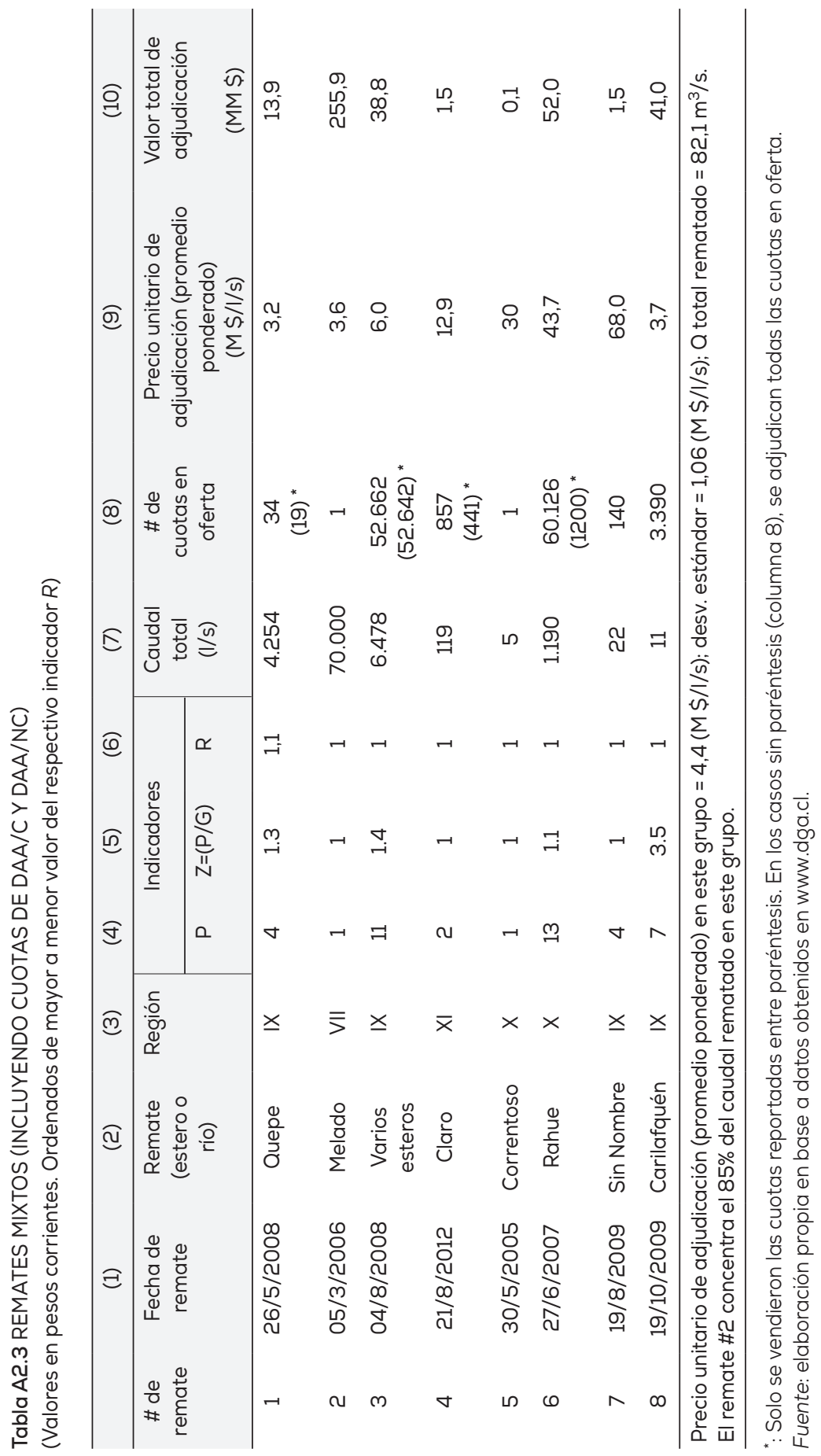




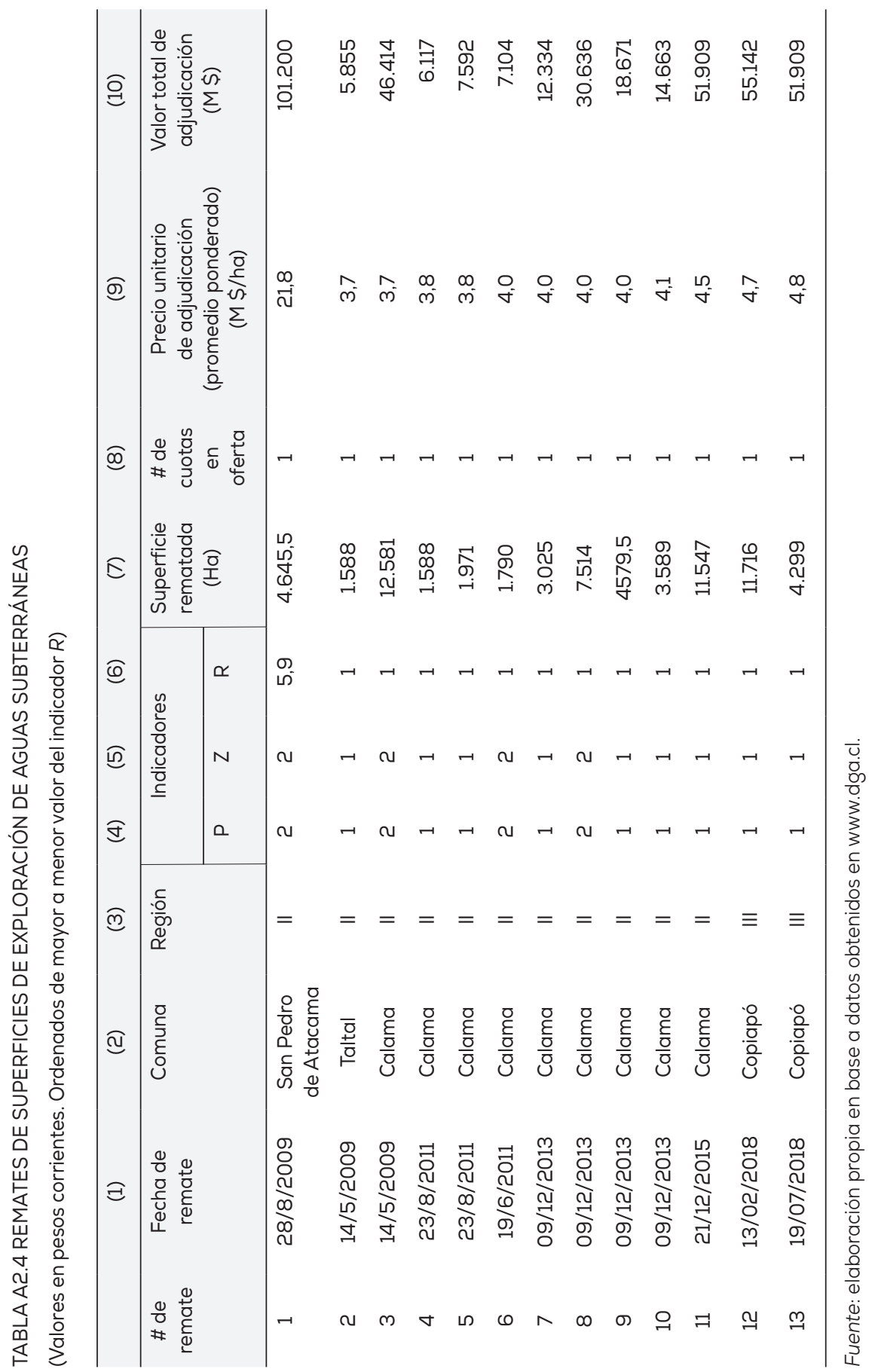

